STABILITY IN PROBABILITY

BANACH CENTER PUBLICATIONS, VOLUME 90

INSTITUTE OF MATHEMATICS

POLISH ACADEMY OF SCIENCES

WARSZAWA 2010

\title{
ON ANALYTICAL PROPERTIES OF GENERALIZED CONVOLUTIONS
}

\author{
ZEEV (VLADIMIR) VOLKOVICH, DVORA TOLEDANO-KITAI and RENATA AVROS \\ Software Engineering Department, ORT Braude College of Engineering \\ Karmiel 21982, Israel \\ E-mail:vlvolkov@braude.ac.il,dvora@braude.ac.il,r_avros@braude.ac.il
}

\begin{abstract}
The paper is, for the most part, devoted to a survey of the analytical properties of generalized convolution algebras and their realizations. This issue appears to be the state of the art until now because intensive research on the generalized convolution and the related models still persists.
\end{abstract}

1. Introduction. The notion of generalized convolutions was introduced by Urbanik in his fundamental paper [43] as a commutative binary operation on probability measures on the non-negative half-line satisfying five axioms. One of them, the so-called 5 -th axiom, was, actually, an analogue of the weak law of large numbers or the central limit theorem for point measures, though the motivating example - the Kingman operation, arising from the study of random walks with spherical symmetry - in no way suggested such an approach. The general and flexible "Urbanik system" encouraged many researchers to investigate this construction and compare it to the known classical analogues. Particularly, the factorizing properties related to the appropriate limit distributions were considered by Bingham [4], [5], Kłosowska [21], Smirnov [42, Jurek [16], 17], and Grishechkin [8]. Class $I_{0}$ for the Kingman convolution was described by Ostrovsky [38], while quasi-stable measures in generalized convolution algebras were considered by Jajte [10, 11]. Generalized Independent Increments Processes indexed by generalized convolutions were studied by Van Thu [36], etc. A detailed analysis of the basic and analytical properties of generalized convolutions was also provided by fundamental studies of Urbanik [44]-[59].

A new approach to analytical description of the Urbanik algebras was proposed by Volkovich 61, 62, 63. A natural method for constructing realizations of general convo-

2010 Mathematics Subject Classification: Primary 60A10; Secondary 60B05.

Key words and phrases: generalized convolutions, stable distributions, hypergroups.

The paper is in final form and no version of it will be published elsewhere. 
lutions concerning the quasi-stable functions and the related $\mathcal{B}$-stable distributions was proposed in [22], 23] and further developed in [47] (see also [64]). Generalizations of this approach make it possible to create and design new probability models, which were introduced by Misiewicz and her colleagues [13, 33, [34. The $V$-infinitely divisible measures introduced by Volkovich [68, [71, [73] were prompted by the general convolutions concept as an attempt to obtain a generalization for the whole real line. Roughly speaking, most of the probability objects for classical convolutions that have been studied so far are investigated, in one or another form, in the framework of the generalized convolutions theory.

The current paper is, for the most part, devoted to a survey of the analytical properties of generalized convolution algebras and their realizations. This issue appears to be the state of the art until now because intensive research on the generalized convolution and related models still persists.

The paper is organized as follows. In Section 2 we present all the notations used in the paper. Section 3 presents the basic definitions and examples of the general convolutions theory. Section 4 is devoted to the discussion of the way in which the classical realization is characterized, namely, to general convolutions stable distributions and to the infinitesimal attributes, related to them. The Banach algebras produced by the general convolutions are presented in section 5. The idea of such an approach was proposed by Volkovich [62], [63]. However, significant development of this idea by Urbanik [51] has led to a major theoretical achievement, which consists in the fact that each convolution is characterized by a function with a measurable kernel. In section 6, non-classical realizations of general convolution are presented, which were obtained by Kucharczak, Urbanik [22, 23], and Volkovich [64], 73] using, mainly, the $\mathcal{B}$-stable distributions. In section 7] the notion of stochastic convolutions, introduced by Volkovich [65, 66, 67, 69, [70, is discussed. This concept has originated from the investigation of various operations on probability measures. The regular generalized convolution algebras provide a perfect example of obtaining significant results on the basis of properly chosen axiomatic system. Other particular cases of this general model are the Levitan convolution, the Lasser polynomial hypergroup convolutions, and the convolutions of Askey and Gasper. In this section, the notion of $V$-infinitely divisible distributions, introduced by Volkovich [68, [71, [73], is also presented. These measures initially appeared in the problems of stochastic centering of the sums of real independent random variables and constitute the basis of the superstable distributions obtained by Zolotarev. The model under consideration is also based on distribution characterizations by means of stochastic properties of linear statistics. New validations of the classical Lévy-Khintchine formula obtained by Volkovich [73], [75], 76] are also presented in this section. In the last section 8, we discuss two open problems of the generalized convolutions theory. The first one concerns the construction of an instructive model of general convolutions on the whole real line, while the second one deals with convolutions representation. In the latter case, the question is whether there exists a regular generalized convolution which cannot be reduced by a power variable exchange to a sub-algebra of symmetric measures on the real line endowed with the regular convolution. 
2. Notations. The following notations are used in this paper:

- $\mathbb{N}=\{1,2, \ldots\}$;

- $\mathbb{R}$ is the real line;

- $\mathbb{R}_{+}=[0, \infty)$;

- $\overline{\mathbb{R}_{+}}$is the compactified half-line $[0, \infty]$;

- $\mathbb{R}^{n}$ is the Euclidean space of dimension $n$.

We consider the above sets as measurable spaces with the natural Borel $\sigma$-algebras.

- $\langle\cdot, \cdot\rangle$ is the inner product.

- $\mathcal{P}(\mathbb{E})$ is the metric space (with weak topology) of all probability measures on a measurable space $\mathbb{E}$ (probability measures are denoted by small Greek letters);

- $\rightarrow$ denotes weak convergence;

- $\mathcal{P}_{+}=\mathcal{P}\left(\mathbb{R}_{+}\right)$;

- $\mathcal{M}(\mathbb{E})$ is the linear space of all $\sigma$-finite signed measures (with weak topology);

- $\mathcal{F}(\mathbb{E})$ is the set of bounded Borel functions defined on $\mathbb{E}$;

- $\mathcal{C}(\mathbb{E})$ is the set of bounded continuous functions defined on $\mathbb{E}$;

- $\mathcal{F}_{+}=\mathcal{F}\left(\mathbb{R}_{+}\right)$;

- $\mathcal{C}_{+}=\mathcal{C}\left(\mathbb{R}_{+}\right)$;

- $\mathcal{M}_{+}=\mathcal{M}\left(\mathbb{R}_{+}\right)$;

- $\delta_{a}$ is the Dirac measure concentrated at the point $a$;

- $\delta_{\infty} \in \mathcal{P}\left(\overline{\mathbb{R}_{+}}\right)$is the Dirac measure concentrated at infinity;

- $\mathbf{m}_{+}$is the Lebesgue measure on $\mathbb{R}_{+}$;

- $T_{a}(\mu)$ is the operator of the multiplicative shift on $\mathcal{P}_{+}$defined as

$$
T_{a} \mu(A)=\left\{\begin{array}{l}
\mu\left(a^{-1} A\right) \text { for } a \neq 0, \\
\delta_{0} \text { for } a=0
\end{array}\right.
$$

- $L_{1}(\mu)$ is the Banach space of all absolutely integrable functions with respect to a measure $\mu$;

- if $\alpha$ and $\beta$ are two measures, then $\alpha \ll \beta$ means that $\alpha$ is absolutely continuous with respect to $\beta$ and $\frac{d \alpha}{d \beta}$ is the Radon-Nikodym derivative of $\alpha$ with respect to $\beta$;

- $\boldsymbol{E}$ is the expected value of a random variable;

- $\otimes$ is the "multiplicative convolution", the operation on $\mathcal{P}_{+}$corresponding to the product of two independent random variables;

- $*_{\alpha}$ is the $\alpha$-convolution;

- $\kappa(\circ)$ is the characteristic exponent of the generalized convolution (g.c.) o;

- $\Gamma$ is the Euler Gamma function.

\section{Generalized convolutions}

3.1. Definitions and examples. Generalized convolutions were introduced by Urbanik [43] as commutative and associative $\mathcal{P}_{+}$-valued binary operations $\circ$ satisfying the following conditions:

1. The measure $\delta_{0}$ is a unit element, i.e.,

$$
\mu \circ \delta_{0}=\mu
$$

for all $\mu \in \mathcal{P}_{+}$. 
2. Linearity:

$$
(c \mu+(1-c) \nu) \circ \eta=c(\mu \circ \eta)+(1-c)(\nu \circ \eta),
$$

for all $0 \leq c \leq 1$ and all $\mu, \nu, \eta \in \mathcal{P}_{+}$.

3. Homogeneity:

$$
T_{a}(\mu \circ \nu)=T_{a} \mu \circ T_{a} \nu
$$

for all $a \in \mathbb{R}_{+}$and all $\mu, \nu \in \mathcal{P}_{+}$.

4. Continuity: if $\mu_{n} \rightarrow \mu$, then

$$
\mu_{n} \circ \nu \rightarrow \mu \circ \nu
$$

for all $\nu \in \mathcal{P}_{+}$.

5. There exists a sequence $c_{1}, c_{2}, \ldots$ of positive numbers such that

$$
T_{c_{n}} \delta_{1}^{\circ(n)} \rightarrow \mu_{0} \neq \delta_{0} .
$$

The power $\delta_{1}^{\circ(n)}$ is taken here in the sense of the operation $\circ$.

Realization, i.e., an explicit example of g.c., can be described in a natural way by means of the following operator defined on $\mathcal{F}_{+}$:

$$
Z^{u} f(v)=\int f(x)\left(\delta_{u} \circ \delta_{v}\right)(d x), \quad f \in \mathcal{F}_{+} .
$$

Obviously, the function

$$
g(f)(u, v):=Z^{u} f(v), u, v \in \mathbb{R}_{+}
$$

belongs to $\mathcal{F}\left(\mathbb{R}_{+} \times \mathbb{R}_{+}\right)$for each $f \in \mathcal{F}_{+}$. We also introduce

$$
Z_{(v)}^{w} Z^{u} f(v)=\int g(f)(u, x)\left(\delta_{w} \circ \delta_{v}\right)(d x) .
$$

Our considerations can be, actually, based only on a part of the properties. First of all, we state the theorem which was proved in several versions in [61], [51, [56].

THEOREM 3.1. Let $\circ$ be a commutative and associative $\mathcal{P}_{+}$-valued binary operation which satisfies the conditions 1-4 above. Then the following holds:

1. $g(f)(u, v) \in \mathcal{C}\left(\mathbb{R}_{+} \times \mathbb{R}_{+}\right)$for each $f \in \mathcal{C}_{+}$;

2. for each $f \in \mathcal{F}_{+}$and every $\mu, \nu \in \mathcal{P}_{+}$:

$$
\iint Z^{u} f(v) \mu(d u) \nu(d v)=\int f(x)(\mu \circ \nu)(d x)
$$

3. for each $f \in \mathcal{F}_{+}$and every $u, v \in \mathbb{R}_{+}$,

$$
Z^{u} f(v)=Z^{v} f(u)
$$

4. for each $f \in \mathcal{F}_{+}$and every $v \in \mathbb{R}_{+}$,

$$
Z^{0} f(v)=f(v)
$$

5. for each $f \in \mathcal{F}_{+}$and every $u, v \in \mathbb{R}_{+}$,

$$
Z_{(v)}^{w} Z^{u} f(v)=Z_{(v)}^{u} Z^{w} f(v) .
$$


Proof. Statements 1, 3, and 4 follow, obviously, from the definition of the operator $Z^{u} f(v)$. To prove statement 2 , we note that, for two discrete measures $\mu$ and $\nu$ represented as

$$
\mu=\sum_{k=1}^{n} \mu_{k} \delta_{a_{k}}, \quad \nu=\sum_{i=1}^{m} \nu_{i} \delta_{b_{i}},
$$

where $a_{k}, b_{i} \in \mathbb{R}_{+}, k=1, \ldots, n, i=1, \ldots, m$, and a function $f \in \mathcal{F}_{+}$, the statement can be explicitly checked.

Next, for any two measures $\mu, \nu \in \mathcal{P}_{+}$, there exist sequences of discrete measures $\left\{\mu_{n}\right\}, n=1,2, \ldots$, and $\left\{\nu_{m}\right\}, m=1,2, \ldots$, such that $\mu_{n} \rightarrow \mu, \nu_{m} \rightarrow \nu$, and

$$
\iint Z^{u} f(v) \mu_{n}(d u) \nu_{m}(d v)=\int f(x)\left(\mu_{n} \circ \nu_{m}\right)(d x), \quad f \in \mathcal{F}_{+} .
$$

Setting here $n, m \rightarrow \infty$, we obtain (2) for each $f \in \mathcal{C}_{+}$. Next, for each $f \in \mathcal{F}_{+}$, we can choose a sequence $\left\{f_{n}\right\}, n=1,2, \ldots$, of functions belonging to $\mathcal{C}_{+}$which converges $(\mu \circ \nu)$-almost everywhere to $f$. According to Lebesgue's bounded convergence theorem,

$$
\lim _{n \rightarrow \infty} \iint Z^{u} f_{n}(v) \mu(d u) \nu(d v)=\iint Z^{x} f(v) \mu(d x) \nu(d v) .
$$

Thus, statement 2 is proved. To prove statement 5 , we consider, for $f \in \mathcal{F}_{+}$,

$$
I(u, v, w)=\int f(x)\left(\delta_{u} \circ \delta_{v} \circ \delta_{w}\right)(d x) .
$$

Due to commutativity and associativity of the operation $\circ$,

$$
I(u, v, w)=\int f(x)\left(\delta_{w} \circ\left(\delta_{u} \circ \delta_{v}\right)\right)(d x)=\int f(x)\left(\delta_{u} \circ\left(\delta_{w} \circ \delta_{v}\right)\right)(d x) .
$$

However, in view of (1),

$$
\int f(x)\left(\delta_{u} \circ\left(\delta_{w} \circ \delta_{v}\right)\right)(d x)=Z_{(v)}^{w} Z^{u} f(v)
$$

and

$$
\int f(x)\left(\delta_{w} \circ\left(\delta_{u} \circ \delta_{v}\right)\right)(d x)=Z_{(v)}^{u} Z^{w} f(v) .
$$

The theorem is proved.

The above theorem allows us to extend g.c. to $\mathcal{M}_{+}$. For this purpose, we can suggest that, in (2), $\mu, \nu \in \mathcal{M}_{+}$and introduce g.c. on $\mathcal{M}_{+}$as

$$
\iint Z^{u} f(v) \mu(d u) \nu(d v)=\int f(x)(\mu \circ \nu)(d x),
$$

where $f \in \mathcal{C}_{+}$. The space $\mathcal{M}_{+}$with this g.c. o will be referred to as a generalized convolution algebra and denoted by $\left(\mathcal{M}_{+}, \circ,+\right)$. Analytical properties of these algebras will be discussed later, while several classical realizations of g.c. in terms of the operator $Z^{u} f(v)$ are listed below.

- $\alpha$-convolution $\left(*_{\alpha}\right)(0<\alpha<\infty)$

$$
Z^{u} f(v)=f\left(\left(u^{\alpha}+v^{\alpha}\right)^{\frac{1}{\alpha}}\right) .
$$


With $\alpha=1$, we obtain the ordinary convolution. Obviously, the sequence $c_{n}=$ $n^{-\frac{1}{\alpha}}(n=1,2, \ldots)$ satisfies condition $(5), \delta_{1}$ being the weak limit of the sequence $T_{c_{n}} \delta_{1}^{\circ(n)}$.

- $\infty$-convolution

$$
Z^{u} f(v)=f(\max (u, v))
$$

Since $\delta_{1}^{\circ(n)}=\delta_{1}(n=1,2, \ldots)$, the sequence $c_{n}=1(n=1,2, \ldots)$ satisfies condition (5). For $\alpha=1$, this operation corresponds to the calculation of the maximum of two independent random variables.

Note that the above two convolutions are not "stochastic" in the sense that the convolution of two point measures is also a point measure.

- $(\alpha, 1)$-convolution $(0<\alpha<\infty)$

$$
Z^{u} f(v)=\frac{f\left(\left(u^{\alpha}+v^{\alpha}\right)^{\frac{1}{\alpha}}\right)+f\left(\left|u^{\alpha}-v^{\alpha}\right|^{\frac{1}{\alpha}}\right)}{2} .
$$

- $(\alpha, \beta)$-convolution $(0<\alpha<\infty, 1<\beta<\infty)$

$$
Z^{u} f(v)=\frac{\Gamma\left(\frac{\beta}{2}\right)}{\Gamma\left(\frac{\beta-1}{2}\right) \sqrt{\pi}} \int_{-1}^{1} f\left(\left(u^{2 \alpha}+v^{2 \alpha}+2 u^{2 \alpha} v^{2 \alpha} z\right)^{\frac{1}{2 \alpha}}\right)\left(1-z^{2}\right)^{\frac{(\beta-3)}{2}} d z .
$$

Setting $c_{n}=n^{-\frac{1}{2 \alpha}}(n=1,2, \ldots)$ for $(\alpha, \beta)$-convolution $(0<\alpha<\infty, 1<\beta<\infty)$, we obtain the probability measure

$$
\mu_{0}(A)=\frac{2 \alpha\left(\frac{\beta}{2}\right)^{\frac{\beta}{2}}}{\Gamma\left(\frac{\beta}{2}\right)} \int_{A} x^{\alpha+\beta-2} \exp \left(-\frac{\beta}{2} x^{2 \alpha}\right) d x
$$

as the weak limit of the sequence $T_{c_{n}} \delta_{1}^{\circ(n)}$. Indeed, this distribution is a version of the well-known Rayleigh's law. For $\alpha=1$, these convolutions were considered by Kingman [20] with regard to random walks with spherical symmetry in an Euclidean space of dimension $\beta$. Urbanik [43] attempted to extend some Kingman's results to g.c.

3.2. Algebra homomorphisms. In the theory of probability, the characteristic functions constitute a crucial tool for solving a great number of practical problems. It appears reasonable to introduce analogous functions in generalized convolution algebras. According to the approach of Urbanik [43, [45], this can be done by means of the so-called algebra homomorphisms.

Definition 3.2. A mapping $h$ of $\mathcal{M}_{+}$into $\mathbb{R}$ is called a homomorphism of the algebra $\left(\mathcal{M}_{+}, \circ,+\right)$ if

- $h(a \mu+b \nu)=a h(\mu)+b h(\nu)$, for all $a, b \in \mathbb{R}$ and all $\mu, \nu \in \mathcal{M}_{+}$;

- $h(\mu \circ \nu)=h(\mu) h(\nu)$, for all $\mu, \nu \in \mathcal{M}_{+}$.

Clearly, each generalized convolution algebra has two trivial homomorphisms: $h \equiv 0$ and $h \equiv 1$. Algebras which admit a non-trivial homomorphism are called weakly-regular. If there is a non-trivial continuous homomorphism, the algebra is called regular. 
THEOREM 3.3. If $h$ is a continuous homomorphism of the algebra $\left(\mathcal{M}_{+}, \circ,+\right)$, then

- $|h(\mu)| \leq 1$ for all $\mu \in \mathcal{P}_{+}$;

- for all $\mu \in \mathcal{M}_{+}$,

$$
h(\mu)=\int h\left(\delta_{x}\right) \mu(d x) .
$$

Proof. Let us prove the first statement by reductio ad absurdum. Suppose that there exists a measure $\mu \in \mathcal{P}_{+}$such that

$$
c=|h(\mu)|>1
$$

Take

$$
\nu_{n}=c^{-2 n} \mu^{\circ(2 n)}+\left(1-c^{-2 n}\right) \delta_{0} .
$$

It is easy to see that

$$
\lim _{n \rightarrow \infty} h\left(\nu_{n}\right)=h\left(\delta_{0}\right) .
$$

On the other hand,

$$
h\left(\mu \circ \delta_{0}\right)=h(\mu)
$$

implies that $\operatorname{ch}\left(\delta_{0}\right)=c$ and

$$
h\left(\delta_{0}\right)=1 .
$$

Therefore,

$$
h\left(\nu_{n}\right)=c^{-2 n} h\left(\mu^{\circ(2 n)}\right)+\left(1-c^{-2 n}\right)=2-c^{-2 n},
$$

which gives

$$
\lim _{n \rightarrow \infty} h\left(\nu_{n}\right)=2 .
$$

However, the last equation is in contradiction with (5) and (6).

Due to linearity of the homomorphism, it is sufficient to check (4) only for $\mu \in \mathcal{P}_{+}$. First of all, it is obvious that, for a discrete measure

$$
\mu=\sum_{k=1}^{n} \mu_{k} \delta_{a_{k}},
$$

we obtain (4). Since the set of all such measures is dense in the sense of weak convergence in $\mathcal{P}_{+}$, (4) holds for all probability measures.

Now, for a homomorphism $h$, we introduce the kernel

$$
\omega_{h}(x)=h\left(\delta_{x}\right) .
$$

Obviously, $\omega_{h}(0)=1$. According to the theorem proved above, this function defines the homomorphism through (4),

$$
Z^{x} \omega_{h}(y)=\omega_{h}(x) \omega_{h}(y)
$$

for all $x, y \in \mathbb{R}_{+}$, and each kernel satisfies this equation. $\omega_{h}(x) \in \mathcal{C}_{+}$if and only if $\circ$ is a regular convolution. 
Let us consider the kernels appearing in the realizations of g.c. presented above.

- $\alpha$-convolution is regular with non-trivial kernels

$$
\omega_{h}(x)=\exp \left(-c x^{\alpha}\right), c>0
$$

- $(\alpha, 1)$-convolution $(0<\alpha<\infty)$ is regular with non-trivial kernels

$$
\omega_{h}(x)=\cos \left(c x^{\alpha}\right), c \neq 0
$$

- $(\alpha, \beta)$-convolution $(0<\alpha<\infty, 1<\beta<\infty)$ is regular with non-trivial kernels

$$
\omega_{h}(x)=\Gamma\left(\frac{\beta}{2}\right)\left(\frac{2}{c x}\right)^{\frac{\beta}{2}-1} J_{\frac{\beta}{2}-1}(c x), c \neq 0,
$$

where $J_{\nu}$ is the Bessel function;

- $\infty$-convolution is weakly-regular with non-trivial kernels

$$
\omega_{h}(x)=\left\{\begin{array}{l}
0 \text { if } x<c, \\
1 \text { otherwise, }
\end{array} \quad c>0 .\right.
$$

Now we can introduce a characteristic function of g.c. for the elements of $\mathcal{M}_{+}$on the basis of a homomorphism $h$ in the form

$$
\Phi_{h}(\mu, t)=\int \omega_{h}(x t) \mu(d x), t \in \mathbb{R}_{+} .
$$

By definition,

- $\Phi_{h}(\mu+\nu, t)=\Phi_{h}(\mu, t)+\Phi_{h}(\nu, t)$, for all $t \in \mathbb{R}_{+}$and all $\mu, \nu \in \mathcal{M}_{+}$;

- $\Phi_{h}(\mu \circ \nu, t)=\Phi_{h}(\mu, t) \Phi_{h}(\nu, t)$, for all $t \in \mathbb{R}_{+}$and all $\mu, \nu \in \mathcal{M}_{+}$;

- $\Phi_{h}\left(T_{a} \mu, t\right)=\Phi_{h}(\mu, a t)$, for all $a, t \in \mathbb{R}_{+}$and all $\mu \in \mathcal{M}_{+}$;

- $\Phi_{h}(\mu, 0)=1$, for all $\mu \in \mathcal{M}_{+}$.

At this stage, we can ask which extension of the transform $\Phi_{h}(\mu, t)$ can reflect the topological properties of the distributions.

THEOREM 3.4. If we suppose that g.c. $\circ$ is regular and there exists a measure $\eta_{0} \in \mathcal{M}_{+}$ such that the family of functions $\Phi_{h}\left(\eta_{0}, c t\right), c \in \mathbb{R}_{+}$is dense in the weak topology in the space $\mathcal{C}_{+}$, then

- each measure $\mu \in \mathcal{M}_{+}$is uniquely determined by its characteristic function $\Phi_{h}(\mu, t)$;

- the sequence $\left\{\mu_{n}\right\}$ of probability measures from $\mathcal{P}_{+}$converges to $\mu \in \mathcal{P}_{+}$if and only if the functions $\Phi_{h}\left(\mu_{n}, t\right)$ uniformly tend to the function $\Phi_{h}(\mu, t)$ in every finite interval.

Proof. Let us suppose that

$$
\Phi_{h}\left(\nu_{1}, t\right)=\Phi_{h}\left(\nu_{2}, t\right)
$$

for all $t \in \mathbb{R}_{+}$. This means that

$$
\int \Phi_{h}\left(\nu_{1}, t u\right) \eta_{0}(d u)=\int \Phi_{h}\left(\nu_{2}, t u\right) \eta_{0}(d u)
$$

for all $t \in \mathbb{R}_{+}$or

$$
\int \eta_{0}(d u) \int \omega_{h}(x t u) \nu_{1}(d x)=\int \eta_{0}(d u) \int \omega_{h}(x t u) \nu_{2}(d x) .
$$


Changing the order of integrations, we obtain

$$
\int \Phi_{h}\left(\eta_{0}, t x\right) \nu_{1}(d x)=\int \Phi_{h}\left(\eta_{0}, t x\right) \nu_{2}(d x)
$$

which implies that $\nu_{1}=\nu_{2}$ because the set $\Phi_{h}\left(\eta_{0}, t x\right)$ is dense in the weak topology in the space $\mathcal{C}_{+}$.

Next, if $\left\{\mu_{n}\right\} \rightarrow \mu$ and $t_{n} \rightarrow t$, then

$$
h\left(T_{t_{n}} \mu_{n}\right) \rightarrow h\left(T_{t} \mu\right) .
$$

In other words, if $\left\{\mu_{n}\right\} \rightarrow \mu$, then the functions $\Phi_{h}\left(\mu_{n}, t\right)$ uniformly tend to the function $\Phi_{h}(\mu, t)$ in every finite interval.

Let us assume that a sequence $\left\{\Phi_{h}\left(\mu_{n}, t\right)\right\}$ uniformly converges to a function $\Phi(t)$ in every finite interval. To show that $\mu_{n}$ weakly converges to a probability measure $\mu$ and that $\Phi(t)=\Phi_{h}(\mu, t)$, it is sufficient to note that the sequence $\left\{\mu_{n}\right\}$ is compact, which means that each subsequence of $\left\{\mu_{n}\right\}$ includes a convergent subsequence. However, it is easy to see that each limit point has the characteristic function $\Phi(t)$. Hence, the sequence $\left\{\mu_{n}\right\}$ is weakly convergent.

Urbanik showed 43, 45, that an important example of the distribution $\eta_{0}$ is provided by the measure $\mu_{0}$ introduced by definition 5 in section 3.1 . The distribution $\mu_{0}$ obeys the following relationship: for all $a, b \in \mathbb{R}_{+}$, there exists $\kappa \in \mathbb{R}_{+} \backslash 0$ such that

$$
T_{a} \mu_{0} \circ T_{b} \mu_{0}=T_{c} \mu_{0},
$$

where $c^{\kappa}=a^{\kappa}+b^{\kappa}$. For a regular g.c., this gives

$$
\Phi_{h}\left(\mu_{0}, t\right)=\exp \left(-c t^{\kappa}\right), c>0 .
$$

Indeed, the measure $\mu_{0}$ is an analogue of a stable distribution defined in the algebras with g.c. Distributions of this kind play a crucial role in the generalized convolutions theory. For this reason, in the next section, we consider stable distribution in detail.

\section{4. o-stable measures and generators}

4.1. o-stable measures. In this section, we present some properties of o-stable measures, which were established in [43], 45], 48], [51]. A measure $\lambda \in \mathcal{P}_{+}$is said to be o-stable if $\lambda \neq \delta_{0}$ and

$$
\lambda=\lim _{n \rightarrow \infty} T_{a_{n}} \nu^{\circ(n)}
$$

for a measure $\nu \in \mathcal{P}_{+}$and a sequence $a_{n}>0$ tending to 0 . The measure $\nu$ is said to belong to the domain of attraction of $\lambda$.

$\boldsymbol{S}$ denotes the set of all o-stable measures. This set is not empty because the measure $\mu_{0}$ introduced in section 3.1, definition 5, is included in $\boldsymbol{S}$. For $0<p \leq \infty$, the set $\boldsymbol{S}_{p}$ comprises all the measures different from $\delta_{0}$ and satisfying, for all $a, b \in \mathbb{R}_{+}$, the equality

$$
T_{a} \lambda \circ T_{b} \lambda=T_{g(a, b)} \lambda
$$


where

$$
g_{p}(a, b)=\left\{\begin{array}{l}
\left(a^{p}+b^{p}\right)^{\frac{1}{p}}, p>0 \\
\max (a, b), p=\infty
\end{array}\right.
$$

It is easy to check that

1. $T_{a}\left(\boldsymbol{S}_{p}\right)=\boldsymbol{S}_{p}, a>0, p>0$;

2. $\boldsymbol{S}_{p_{1}} \cap \boldsymbol{S}_{p_{2}}=\emptyset$ for $p_{1} \neq p_{2}$;

3. $\boldsymbol{S}_{p} \subset \boldsymbol{S}$ for $0<p<\infty$, which follows from the equation

$$
T_{a_{n}} \lambda^{\circ(n)}=\lambda,
$$

where $a_{n}=n^{-\frac{1}{p}}$;

4. $\boldsymbol{S}_{\infty} \neq \emptyset$ if and only if g.c. is $\infty$-convolution. Obviously, for $\infty$-convolution, $\boldsymbol{S}_{\infty}=$ $\left\{\delta_{a}: a>0\right\}=S$

5. $\lambda \in \boldsymbol{S}_{p}$ does not have an atom at the origin.

Proof. To prove assertion 5 , we take $\lambda \in \boldsymbol{S}_{p}$. By assertion 4 , we can suppose that $p<\infty$. In this case,

$$
\lambda=c \delta_{0}+(1-c) \beta
$$

where $0 \leq c<1$ and $\beta \in \mathcal{P}_{+}$. Thus

$$
\lambda \circ T_{n} \lambda=T_{b_{n}} \lambda=c \delta_{0}+(1-c) T_{b_{n}} \beta,
$$

where $b_{n}=\left(1+n^{p}\right)^{\frac{1}{p}}$. It follows that

$$
\lim _{n \rightarrow \infty}\left(\lambda \circ T_{n} \lambda\right)=c \delta_{0}+(1-c) \delta_{\infty} .
$$

Alternatively,

$$
\lambda \circ T_{n} \lambda=c^{2} \delta_{0}+c(1-c) T_{n} \beta+c(1-c) \beta+\left(1-c^{2}\right)\left(\beta \circ T_{n} \beta\right)
$$

and, in view of

$$
\lim _{n \rightarrow \infty} T_{n} \beta=\lim _{n \rightarrow \infty}\left(\beta \circ T_{n} \beta\right)=\delta_{\infty}
$$

we obtain

$$
\lim _{n \rightarrow \infty}\left(\lambda \circ T_{n} \lambda\right)=c \delta_{0}+c(1-c) \beta+(1-c) \delta_{\infty} .
$$

The comparison of $\sqrt{12}$ and 13 shows that $c=c^{2}$, which yields $c=0$.

For each $\alpha<\infty$ there exists a power variable substitution under which the convolution $*_{\alpha}$ transforms into $*_{1}$. Based on this fact, we can conclude that, in this case, $\boldsymbol{S}_{p}=\emptyset$ for $p>\alpha$ and the density of each distribution which belongs to $\boldsymbol{S}_{p}, p<\alpha$, is expressed as

$$
\gamma_{p, \alpha}(E)=\alpha \int_{E} R_{\frac{p}{\alpha}}\left(x^{-\alpha p}\right) \frac{d x}{x},
$$

where $R_{\frac{p}{\alpha}}$ is an entire function (see, for example, [77]). Moreover, if $\lambda \in \boldsymbol{S}_{p}$ for a g.c. o, then, for all $\mu, \nu \in \mathcal{P}_{+}$,

$$
(\lambda \otimes \mu) \circ(\lambda \otimes \nu)=\lambda \otimes\left(\mu *_{p} \nu\right),
$$


where $\otimes$ is the "multiplicative convolution". To prove the above relationship, by theorem 3.1 and $(11)$, we obtain the following relation:

$$
\begin{aligned}
(\lambda \otimes \mu) \circ(\lambda \otimes \nu) & =\iint\left(\lambda \otimes \delta_{u}\right) \circ\left(\lambda \otimes \delta_{v}\right) \mu(d u) \nu(d v) \\
& =\iint \lambda\left(\mu *_{p} \nu\right) \mu(d u) \nu(d v) \\
& =\lambda \otimes\left(\mu *_{p} \nu\right) .
\end{aligned}
$$

Let $X_{p}$ be a random variable having the probability distribution $\gamma_{p, 1}$. For any $p$ $(0<p<\infty)$ and $q(0<q<p)$, denote by $\pi_{p, q}$ the probability distribution of the random variable $Y=X_{\frac{q}{p}}^{\frac{1}{p}}$.

Lemma 4.1. Let $\lambda \in \boldsymbol{S}_{p}(0<p<\infty)$. Then, for any $q(0<q<p)$,

- $\gamma=\lambda \otimes \pi_{p, q} \in \boldsymbol{S}_{q}$;

- the measure $\gamma$ has almost everywhere positive density;

- $\lambda \otimes \pi_{p, q} \rightarrow \lambda$ if $p \rightarrow q$.

Proof. According to the definition of $\eta_{p, 1}$, we get

$$
\exp \left(-t^{\frac{q}{p}}\right)=\int \exp (-t x) \eta_{\frac{q}{p}, 1}(d x), t \in \mathbb{R}_{+}
$$

and the density of $\pi_{p, q}$ is

$$
\rho\left(\pi_{p, q}\right)(x)=p R_{\frac{q}{p}}\left(x^{-q}\right) \frac{d x}{x} .
$$

Let us calculate

$$
\Phi_{p}\left(\pi_{p, q}, t\right)=p \int \exp \left(-(t x)^{p}\right) R_{\frac{q}{p}}\left(x^{-q}\right) \frac{d x}{x}
$$

through the variable substitution $x \rightarrow x^{\frac{1}{p}}$ :

$$
\Phi_{p}\left(\pi_{p, q}, t\right)=\int \exp \left(-t^{p} x\right) R_{\frac{q}{p}}\left(x^{-\frac{q}{p}}\right) \frac{d x}{x}=\exp \left(-t^{q}\right) .
$$

It follows from the above that, for all $a, b \in \mathbb{R}_{+}$, the equality

$$
\left(T_{a} \pi_{p, q}\right) *_{p}\left(T_{b} \pi_{p, q}\right)=T_{g_{q}(a, b)} \pi_{p, q}
$$

holds. Furthermore, employing (14), we obtain for all $a, b \in \mathbb{R}_{+}$

$$
\begin{aligned}
T_{a}(\gamma) \circ T_{a}(\gamma) & =\left(\lambda \otimes T_{a}\left(\pi_{p, q}\right)\right) \circ\left(\lambda \otimes T_{b}\left(\pi_{p, q}\right)\right) \\
& =\lambda \otimes\left(T_{a}\left(\pi_{p, q}\right) *_{p} T_{b}\left(\pi_{p, q}\right)\right) \\
& =\lambda \otimes T_{g_{q}(a, b)} \pi_{p, q}=T_{g_{q}(a, b)}\left(\lambda \otimes \pi_{p, q}\right)=T_{g_{q}(a, b)}(\lambda) .
\end{aligned}
$$

Thus $\gamma \in \boldsymbol{S}_{q}$.

Obviously, $\gamma$ has almost everywhere positive density because so does $\pi_{p, q}$. The last assertion of Lemma 4.1 follows from the fact that $\pi_{p, q} \rightarrow \delta_{1}$ if $p \rightarrow q$.

Note that if $\boldsymbol{S}_{\infty} \neq \emptyset$, then, according to the above property 5 , g.c. coincides with $\infty$-convolution and, for any $(0<q<\infty)$, the Weibull-Gnedenko distribution with the density

$$
\rho_{q}(x)=q x^{-(q+1)} \exp \left(-x^{-q}\right)
$$


belongs to $\boldsymbol{S}_{q}$. If $\boldsymbol{S}_{p} \neq \emptyset(0<p<\infty)$, then $\boldsymbol{S}_{q} \neq \emptyset$ for all $(0<q \leq p)$ (see Lemma 4.1), and, consequently, for each $0<q<p$ there exists a distribution $\lambda_{p}$ which has almost everywhere positive density. Such value of $p$ exists for any g.c.

Some main properties of o-stable distributions can be summarized in the following form:

- For any g.c. $\circ$, the following statement is true:

$$
\boldsymbol{S}=\bigcup_{p} \boldsymbol{S}_{p} .
$$

- For every g. c. $\circ$, there exists an index $\kappa(0<\kappa \leq \infty)$ such that

$-\boldsymbol{S}_{p}=\emptyset$ for $p>\kappa$ and $\boldsymbol{S}_{p} \neq \emptyset$ for all $0<q \leq \kappa$;

- For each $0<p<\kappa$, there exists a distribution $\lambda_{p}$ having almost everywhere positive density.

The index $\kappa$ is called the characteristic exponent of the g.c. $\circ$ and denoted by $\kappa(\circ)$.

- For any measure belonging to the domain of attraction of a measure from $\boldsymbol{S}_{p}(0<p$ $<\infty)$, the moments

$$
M_{p}=\int u^{q} \mu(d u)
$$

are finite for all $0<q<p$ and, for all $s>\frac{1}{p}$,

$$
\lim _{n \rightarrow \infty} T_{n^{-s}} \mu^{\circ(n)}=\delta_{0} .
$$

4.2. Infinitesimal characteristics. The approach described below is based on the application of the generalized shift operators (g.s.o.) theory relying on theorem 3.1, which states that, for every $u, v, w \in \mathbb{R}_{+}$and $f \in \mathcal{F}_{+}$, the relationship

$$
Z_{(v)}^{w} Z^{u} f(v)=Z_{(v)}^{u} Z^{w} f(v)
$$

holds. This relationship, together with

$$
Z^{0} f(v)=f(v), v \in \mathbb{R}_{+},
$$

shows that the set of operators $Z^{u}, u \in \mathbb{R}_{+}$, constitutes a family of g.s.o.

Levitan 28 constructed a convolution on $\mathcal{P}_{+}$using infinitesimal operators of a g.s.o. family in the following way. Let $a(x)$ and $b(x)$ be real-valued positive continuous functions on $\mathbb{R}_{+}$. Denote by $\omega(x, \lambda), \lambda \in \mathbb{R}$, the solution of the problem

$$
\begin{gathered}
S_{(x)}(y)=b(x) y^{\prime \prime}(x)+a(x) y^{\prime}(x)=-\lambda y, \\
y(0)=1, y^{\prime}(0)=0 .
\end{gathered}
$$

The convolution is, actually, introduced via equation (2), where, for each two times differentiable function from $\mathcal{C}_{+}$, the operators $g(u, v)=Z^{u} f(v)$ are defined as solutions with several additional conditions:

$$
\begin{gathered}
S_{(u)}(g)=S_{(v)}(g), \\
g(u, 0)=f(u),\left.\frac{\partial g}{\partial v}\right|_{u=0}=0 .
\end{gathered}
$$


Here $|\omega(x, \lambda)| \leq 1$ for all values of $x$ and $\lambda$ and the integral transform

$$
\Phi(\mu, \lambda)=\int \omega(x, \lambda) \mu(d x), \mu \in \mathcal{P}_{+}
$$

plays the role of the generalized characteristic function.

It is interesting to note that the present results were previously obtained in [27] for the particular case of

$$
S_{(x)}(y)=y^{\prime \prime}(x)+\frac{\beta-1}{x} y^{\prime}(x), \beta \geq 1 .
$$

Here $\omega(x, \lambda)$ coincides with the kernel of the characteristic function of $(1, \beta)$-convolution. Thus, the Levitan convolutions were introduced earlier in a proper analytical way based on the realizations which were later shown to be the classical realizations of the Urbanik generalized convolutions, introduced on the basis of their probabilistic properties. It would be natural to ask if g.c. algebras could be defined by means of generators similar to (15). Such an approach was considered by Volkovich [61.

We say that a family of measures $\left\{\mu_{t}\right\} \in \mathcal{P}_{+}, t \in \mathbb{R}_{+}$, is a g. c. semigroup (or a o-semigroup) if the following conditions are satisfied:

$$
\begin{aligned}
\mu_{t} \circ \mu_{s} & =\mu_{t+s}(t, s \geq 0) ; \\
\lim _{t \rightarrow 0} \mu_{t} & =\delta_{0} .
\end{aligned}
$$

It follows from the above that $\mu_{0}=\delta_{0}$. A o-semigroup induces a time-homogeneous o-independent increments process, which is often called a o-Lévy process (see, for example, [36]). Such a process can be naturally constructed by means of the o-stable distributions.

Let $\lambda_{p}$ be a o-stable distribution corresponding to a power $p<\infty$. Introduce the laws

$$
\lambda_{p, t}=T_{t^{\frac{1}{p}}} \lambda_{p}, t \in \mathbb{R}_{+} .
$$

Obviously,

$$
\lambda_{p, t_{1}} \circ \lambda_{p, t_{2}}=\lambda_{p}, t_{1}+t_{2}
$$

for all $t_{1}, t_{2} \in \mathbb{R}_{+}$.

Let $\left\{\mu_{t}\right\}$ be a o-semigroup. Consider the space $\mathcal{L}\left(\mu_{t}\right)$ of the Borel functions on $\mathbb{R}_{+}$ satisfying, for $u \in \mathbb{R}_{+}$, the condition

$$
f \in L_{1}\left(\delta_{u} \circ \mu_{t}\right) .
$$

Clearly, $\mathcal{C}_{+} \subset \mathcal{L}\left(\mu_{t}\right)$, for each measure $\mu_{t}$. Introduce a family of operators defined on $\mathcal{L}\left(\mu_{t}\right)$ :

$$
\boldsymbol{P}_{t}(f)(u)=\int f(x)\left(\delta_{u} \circ \mu_{t}\right)(d x) .
$$

It is easy to check that this family gives an operator semigroup

$$
\boldsymbol{P}_{t_{2}}\left(\boldsymbol{P}_{t_{1}}(f)(u)\right)=\boldsymbol{P}_{t_{1}+t_{2}}(f)(u) .
$$

Clearly,

$$
\lim _{t \rightarrow 0} \boldsymbol{P}_{t}(f)(u)=f(u)
$$


for all functions $f \in \mathcal{C}_{+}$, for each $u \in \mathbb{R}_{+}$. Denote by $S\left(\lambda_{p}, t\right)$ the infinitesimal operator of $\boldsymbol{P}_{t}(f)$ in $\mathcal{L}\left(\mu_{t}\right)$ with the domain $\operatorname{Dom}\left(S\left(\lambda_{p}, t\right)\right)$. The following statement was shown to be valid [61]:

If $f \in \operatorname{Dom}\left(S\left(\lambda_{p}, t\right)\right)$, then the relationship

$$
g(u, v)=Z^{u} f(v)
$$

holds if and only if

$$
S\left(\lambda_{p, t}\right)_{(u)}(g(u, v))=S\left(\lambda_{p, t}\right)_{(v)}(g(u, v))
$$

and

$$
g(0, v)=f(v), g(u, 0)=f(u) .
$$

This proposition shows that every g.c. is uniquely defined by each of the operators $S\left(\lambda_{p, t}\right)$. Such an approach coincides with Levitan's approach presented in (15). The description of the intersection of these convolutions construction was given in [1].

THEOREM 4.2. If there exists $p$ such that $S\left(\lambda_{p, t}\right)$ is a differential operator, then the convolution is either $\alpha$-convolution $(0<\alpha<\infty)$ or $(\alpha, \beta)$-convolution $(0<\alpha<\infty$, $1 \leq \beta<\infty)$ and, for $\alpha=1$, the operator $S\left(\lambda_{p}, t\right)$ is represented as

- $S\left(\lambda_{p, t}\right)(f)(x)=\frac{d f}{d x}, \alpha$-convolution;

- $S\left(\lambda_{p, t}\right)(f)(x)=\frac{d^{2} f}{d x^{2}},(\alpha, 1)$-convolution;

- $S\left(\lambda_{p, t}\right)(f)(x)=\frac{d^{2} f}{d x^{2}}+\frac{(\beta-1)}{2 x} \frac{d f}{d x},(\alpha, \beta)$-convolution.

\section{Banach algebras associated with generalized convolutions}

5.1. Generalized convolutions of functions. It is known that the Banach algebras are an appropriate tool for the study of the analytical properties of the probability distribution on locally compact groups. It appears that the construction of a similar instrument to be used in algebras with g.c. would be quite natural. This approach was first suggested by Volkovich [62, 63], the crucial notions of the concept being the o-quasi-invariant measures. A similar technique was also discussed later [37.

Definition 5.1. A measure $\eta \in \mathcal{P}_{+}$is called o-quasi-invariant if

$$
\mu \circ \delta_{u} \ll \eta
$$

for all $\mu \ll \eta$ and $u \in \mathbb{R}_{+}$.

So, if $\eta$ is a o-quasi-invariant measure, then, according to theorem 3.1, for two measures $\alpha, \beta \in \mathcal{M}_{+}$absolutely continuous with respect to the measure $\eta$, we get

$$
(\alpha \circ \beta)(E)=\iint\left(\delta_{u} \circ \delta_{v}\right)(E) \alpha(d u) \beta(d v)=\int\left(\alpha \circ \delta_{u}\right)(E) \beta(d u) \ll \eta .
$$

Thus, the measure $(\alpha \circ \beta)$ is absolutely continuous with respect to the measure $\eta$. Moreover, if we introduce

$$
\widehat{\eta}=\delta_{0}+\eta
$$

then, for two measures $\alpha, \beta \ll \widehat{\eta}$, we obtain

$$
(\alpha \circ \beta)(E)=a b \delta_{0}(E)+a(1-b) \beta^{\prime}(E)+(1-a) b \alpha^{\prime}(E)+\left(\alpha^{\prime} \circ \beta^{\prime}\right)(E),
$$


where

$$
\alpha=a \delta_{0}+(1-a) \alpha^{\prime}, \beta=b \delta_{0}+(1-b) \beta^{\prime},
$$

$0 \leq a, b \leq 1$ and $\alpha^{\prime}, \beta^{\prime} \ll \eta$. Now we can define g.c. for the elements of $L_{1}(\widehat{\eta})$. For two functions $f_{1}, f_{2} \in L_{1}(\widehat{\eta})$, we consider $\widehat{\eta}\left(f_{1}\right) \circ \widehat{\eta}\left(f_{2}\right)$ for

$$
\widehat{\eta}\left(f_{i}\right)(E)=\int_{E} f_{i}(u) \widehat{\eta}(d u), i=1,2 \text {. }
$$

It is clear that

$$
\widehat{\eta}\left(f_{1}\right) \circ \widehat{\eta}\left(f_{2}\right)(E)=\int\left(\widehat{\eta}\left(f_{2}\right) \circ \delta_{v}\right)(E) f_{1}(v) \widehat{\eta}(d v)=\int f_{1}(v) \widehat{\eta}(d v) \int_{E} A^{v} f_{2}(u) \widehat{\eta}(d u),
$$

where

$$
A^{v} f_{2}(u)=\frac{d\left(\widehat{\eta}\left(f_{2}\right) \circ \delta_{v}\right)(u)}{d \widehat{\eta}} .
$$

Based on the above, we introduce g.c. for the elements of $L_{1}(\widehat{\eta})$ as

$$
\left(f_{1} \circ f_{2}\right)(u)=\int f_{1}(v) A^{v} f_{2}(u) \widehat{\eta}(d v) .
$$

In addition, it can be seen that, for any $g \in L_{\infty}(\widehat{\eta})$,

$$
\int Z^{v} g(u) f(u) \widehat{\eta}(d u)=\int g(u)\left(\widehat{\eta}(f) \circ \delta_{v}\right)(d u)=\int g(u) A^{v} f(u) \widehat{\eta}(d u) .
$$

Next, we introduce the symmetric functional algebra

$$
\mathfrak{L}=\left(L_{1}(\widehat{\eta}),+, \circ, \sim\right),
$$

where the involution is $\widetilde{x}=\bar{x}$ for $x \in L_{1}(\widehat{\eta})$ and the bar denotes the complex conjugate. It is easy to check that $\mathfrak{L}$ is a symmetric, commutative, and associative Banach algebra with the unit $\delta_{0}$. It is well-known (see e.g., [35]) that the maximal ideals of the convolution algebras on locally compact groups play a crucial role in harmonic analysis on groups.

By analogy, it appears natural to study the maximal ideals of the newly constructed algebra. Let $\widehat{\mathfrak{M}}$ be the set of all maximal ideals of $\mathfrak{L}$ different from $L_{1}(\widehat{\eta})$. Take a maximal ideal $\mathfrak{M} \in \widehat{\mathfrak{M}}$ and consider the corresponding homomorphism $h(\mathfrak{M})$ of $\mathfrak{L}$ :

$$
h(\mathfrak{M})(x)=\int \omega_{\mathfrak{M}}(v) x(v) \widehat{\eta}(x)(d v),
$$

where $\omega_{\mathfrak{M}} \in \mathcal{F}_{+}$. It can be seen that, for $x, y \in L_{1}(\widehat{\eta})$,

$$
\begin{aligned}
h(\mathfrak{M})(x \circ y) & =\iint \omega_{\mathfrak{M}}(v) x(u) A^{v} y(u) \widehat{\eta}(x)(d v) \widehat{\eta}(x)(d u) \\
& =\iint Z^{v} \omega_{\mathfrak{M}}(v) \widehat{\eta}(x)(x)(d u) \widehat{\eta}(x)(y)(d v) .
\end{aligned}
$$

On the other hand,

$$
h(\mathfrak{M})(x \circ y)=h(\mathfrak{M})(x) h(\mathfrak{M})(y)=\int \omega_{\mathfrak{M}}(u) \widehat{\eta}(x)(x)(d u) \int \omega_{\mathfrak{M}}(v) \widehat{\eta}(x)(y)(d v) .
$$

Below, some properties of the kernels $\omega_{\mathfrak{M}}(u)$ are summarized.

1. $Z^{v} \omega_{\mathfrak{M}}(u)=\omega_{\mathfrak{M}}(v) \omega_{\mathfrak{M}}(u)$ holds $\widehat{\eta} \times \widehat{\eta}$ almost surely. In other words, the function $\omega_{\mathfrak{M}}(v)$ satisfies the kernel equation $(7) \widehat{\eta} \times \widehat{\eta}$ almost surely. 
2. $\omega_{\mathfrak{M}}(0)=1$. This can be proved by setting $\widehat{\eta}(x)=\widehat{\eta}(y)=\delta_{0}$. It follows from the previous equation that

$$
\omega_{\mathfrak{M}}^{2}(0)=\omega_{\mathfrak{M}}(0)
$$

Note that $\omega_{\mathfrak{M}}(0) \neq 0$ because, otherwise,

$$
h(\mathfrak{M})\left(x \circ \delta_{0}\right)=h(\mathfrak{M})(x)=0
$$

for each $x \in L_{1}(\widehat{\eta})$, which contradicts $\mathfrak{M} \neq L_{1}(\widehat{\eta})$.

3. $\left\|\omega_{\mathfrak{M}}\right\|_{\infty}=1$. According to 16 ,

$$
\|h(\mathfrak{M})\|=\left\|\omega_{\mathfrak{M}}\right\|_{\infty} .
$$

On the other hand, it is well known (see, for example, 35]) that $\left\|\omega_{\mathfrak{M}}\right\|_{\infty} \leq 1$; yet $\omega_{\mathfrak{M}}(0)=1$.

4. Suppose that, for each $y \in L_{1}(\widehat{\eta})$,

$$
\lim _{v_{n} \rightarrow v} A^{v_{n}} y(u)=A^{v} y(u)
$$

in the $L_{1}(\widehat{\eta})$ norm, which results in $\omega_{\mathfrak{M}} \in \mathcal{C}_{+}$. In view of the previous assertion, we must only make sure that the kernel is continuous.

Let us take $y \in L_{1}(\widehat{\eta})$ such that $h(\mathfrak{M})(y)=1$. We can see that

$$
\begin{aligned}
& \left|\omega_{\mathfrak{M}}\left(v_{n}\right)-\omega_{\mathfrak{M}}(v)\right|=\left|\int\left(Z^{v_{n}} \omega_{\mathfrak{M}}(u)-Z^{v} \omega_{\mathfrak{M}}(u)\right) y(u) \eta(d u)\right| \\
& \quad=\left|\int \omega_{\mathfrak{M}}(u)\left(A^{v_{n}} y(u)-A^{v} y(u)\right) \eta(d u)\right| \leq \int\left|A^{v_{n}} y(u)-A^{v} y(u)\right| \eta(d u) \rightarrow 0
\end{aligned}
$$

if $v_{n} \rightarrow v$.

Although the concept of the o-quasi-invariant measures was introduced in [62, 63], this approach was significantly extended and generalized by Urbanik [51, [54] in the following way.

Introduce the measure

$$
\mathbf{m}_{0}=\delta_{0}+\mathbf{m}_{+}
$$

and denote by $V_{0}$ the subset of $\mathcal{M}_{+}$containing all the measures which are absolutely continuous with respect to the measure $\mathbf{m}_{0}$.

LEMMA 5.2. The space $V_{0}$ is closed under g.c.

Proof. Let us take $\lambda \in \boldsymbol{S}_{p}(0<p<\infty)$ having almost everywhere positive density. We get

$$
T_{2^{\frac{1}{p}}} \lambda(E)=(\lambda \circ \lambda)(E)=\iint\left(\delta_{u} \circ \delta_{v}\right)(E) \lambda(d u) \lambda(d v) .
$$

Suppose that $\mathbf{m}_{0}(E)=0$. Since $\lambda \ll \mathbf{m}_{+}$, we have $T_{2^{\frac{1}{p}}} \lambda(E)=0$, which implies that $\left(\delta_{u} \circ \delta_{v}\right)(E)=0$ for $(u, v) \in \mathbb{R}_{+} \times \mathbb{R}_{+} \lambda \times \lambda$-almost surely. Thus,

$$
(\alpha \circ \beta)(E)=\iint\left(\delta_{u} \circ \delta_{v}\right)(E) \alpha(d u) \beta(d v)=0
$$


for any two measures $\alpha, \beta \ll \mathbf{m}_{+}$. Next, let us consider two measures which belong to $V_{o}$ :

$$
\widehat{\alpha}=a \delta_{0}+(1-a) \alpha, \widehat{\beta}=b \delta_{0}+(1-b) \beta,
$$

where $\alpha, \beta \ll \mathbf{m}_{+}$. It is easy to see that

$$
(\widehat{\alpha} \circ \widehat{\beta})(E)=a b \delta_{0}(E)+a(1-b) \beta(E)+b(1-a) \alpha(E)+(1-a)(1-b)(\alpha \circ \beta)(E) .
$$

If $\mathbf{m}_{0}(E)=0$, then

$$
\delta_{0}(E)=0, \alpha(E)=0, \beta(E)=0
$$

and, in view of 17$),(\widehat{\alpha} \circ \widehat{\beta})(E)=0$.

This fundamental lemma, established in [51, makes it possible to construct, in a similar way, the normed ring $\mathfrak{L}=\left(L_{1}\left(\mathbf{m}_{0}\right),+, \circ, \sim\right)$. Below, we briefly describe the analytical results obtained in the paper on the basis of the algebra ideals properties.

5.2. Maximal ideals. Our purpose is to design an analogue of the characteristic function $(9)$ in the form

$$
\Phi_{\mathfrak{M}}(\mu, t)=\int \omega_{\mathfrak{M}}(t u) \mu(d u), \quad t \in \mathbb{R}_{+}
$$

where $\mathfrak{M} \in \widehat{\mathfrak{M}}$.

In what follows, $\mathfrak{M}^{s}$ denotes the subset of the maximal ideals corresponding to real-valued kernels. This set consists of the symmetric ideals obeying the condition $\widetilde{x}(\mathfrak{M})=\overline{x(\mathfrak{M})}$. Two trivial symmetric ideals are

$$
x\left(\mathfrak{M}_{0}\right)=\int x(v) \mathbf{m}_{0}(d v), \quad x\left(\mathfrak{M}_{\infty}\right)=x(\{0\}) .
$$

We also introduce

$$
\mathfrak{M}_{+}^{s}=\mathfrak{M}^{s} \backslash\left\{\mathfrak{M}_{0}, \mathfrak{M}_{\infty}\right\}
$$

and

$$
\mathfrak{M}_{\lambda}=\left\{\mathfrak{M} \mid\left(T_{2} \lambda_{p}-\lambda_{p}\right) \neq 0\right\}
$$

for an absolutely continuous, with respect to $\mathbf{m}_{0}$, measure $\lambda_{p} \in \boldsymbol{S}$. In this case, $\mathfrak{M}_{\lambda_{p}} \neq \emptyset$, $\mathfrak{M}_{0}, \mathfrak{M}_{\infty} \notin \mathfrak{M}_{\lambda_{p}}$ and there exists a complex constant $c(\mathfrak{M})$ with $\operatorname{Re}(c(\mathfrak{M}))>0$ such that

$$
\Phi_{\mathfrak{M}}\left(\lambda_{p}, t\right)=\exp \left(-c(\mathfrak{M}) t^{p}\right)
$$

for all $t \in \mathbb{R}_{+}$and $\mathfrak{M} \in \mathfrak{M}_{\lambda_{p}}$. Moreover, it is possible to prove that, for each $\lambda_{p}$,

$$
\mathfrak{M}^{s} \cap \mathfrak{M}_{\lambda_{p}} \neq \emptyset
$$

and the set $\mathfrak{M}_{+}^{s}$ is not empty. This means that each g.c. possesses a non-trivial real-valued kernel of the $\omega_{\mathfrak{M}}(u)$ kind.

THEOREM 5.3. Let $\omega_{\mathfrak{M}^{(0)}}(u), \mathfrak{M}^{(0)} \in \mathfrak{M}_{+}^{s}$, be an arbitrary kernel. Then, for any $\mathfrak{M}^{(1)} \in$ $\mathfrak{M}_{+}^{s}$, there exists a value $a \in \mathbb{R}_{+} \backslash 0$ such that, for all $u \in \mathbb{R}_{+}$,

$$
\omega_{\mathfrak{M}}(u)=\omega_{\mathfrak{M}(0)}(a u)
$$

$\mathbf{m}_{0}$-almost everywhere, the correspondence between $\mathbb{R}_{+} \backslash 0$ and $\mathfrak{M}_{+}^{s}$ being one-to-one. 
The following analogue of the classical Bochner theorem can be considered as a direct application of the above results. A continuous linear functional $l$ on $\mathfrak{L}$ is said to be positive if

$$
l(x \circ \bar{x}) \geq 0
$$

for all $x \in \mathfrak{L}$.

THEOREM 5.4. Each positive continuous linear functional l on $\mathfrak{L}$, for any kernel $\omega_{\mathfrak{M}}(u)$, $\mathfrak{M} \in \mathfrak{M}_{+}^{s}$, has the representation

$$
l(\alpha)=\int_{\overline{\mathbb{R}_{+}}} \omega_{\mathfrak{M}}(t u) \sigma(d u)
$$

where $\sigma$ is a bounded Borel measure on $\overline{\mathbb{R}_{+}}$.

5.3. Weak characteristic functions. The concept of a characteristic function for g.c. was discussed in section 3.2. In particular, we saw that a g.c. admits a characteristic function if and only if it is regular. Thus, there exists a non-constant continuous homomorphism from the g.c. algebra into the real numbers algebra. Although not every g.c. is regular, the real-valued kernels considered in the previous section make it possible to extend the characteristic function concept to all generalized convolutions.

We say that the g.c. $\circ$ admits a weak characteristic function if there exists a one-to-one correspondence $\widehat{h}$ between $\mathcal{P}_{+}$and $\mathcal{F}_{+}$such that

1. $\widehat{h}(\mu)$ is continuous if $\mu \in \mathcal{P}_{+} \cap V_{0}$;

2. $\widehat{h}(c \mu+(1-c) \nu)=c \widehat{h}(\mu)+(1-c) \widehat{h}(\nu)$ for all $0 \leq c \leq 1$ and all $\mu, \nu \in \mathcal{P}_{+}$;

3. $\widehat{h}(\mu \circ \nu)=\widehat{h}(\mu) \widehat{h}(\nu)$ for all $\mu, \nu \in \mathcal{P}_{+}$;

4. $\widehat{h}\left(T_{a} \mu\right)(t)=\widehat{h}(\mu)(a t)(a>0)$;

5. $\mathbf{m}_{0}$-almost everywhere for every $\mu \in \mathcal{P}_{+}$

$$
\lim _{a \rightarrow 0} \widehat{h}\left(\eta_{a} \circ \mu\right)=\widehat{h}(\mu)
$$

and the pointwise convergence $\widehat{h}\left(\eta_{a} \circ \mu_{n}\right) \rightarrow \widehat{h}\left(\eta_{a} \circ \mu\right)$ on $\mathbb{R}_{+}$for all $a>0$ is equivalent to the convergence $\mu_{n} \rightarrow \mu$.

Note that all the equalities considered here are true $\mathbf{m}_{0}$-almost everywhere.

To begin with, we list some properties of weak characteristic functions which directly follow from their definition.

1. $\widehat{h}\left(\delta_{0}\right) \equiv 1$;

2. $\widehat{h}(\mu)(0)=1$ for all $\mu \in \mathcal{P}_{+}$;

3. $\|\widehat{h}(\mu)\|_{\infty}=1$ for all $\mu \in \mathcal{P}_{+}$.

To prove the last assertion we obviously conclude from the two previous ones that

$$
\|\widehat{h}(\mu)\|_{\infty} \geq 1 \text {. }
$$

Suppose that $\|\widehat{h}(\mu)\|_{\infty}=c>1$. Then

$$
\left\|\frac{\widehat{h}^{2}(\mu)}{c^{2}-\widehat{h}^{2}(\mu)}\right\|_{\infty}=\infty .
$$


Introduce

$$
\lambda=\left(c^{2}-1\right) \sum_{n=1}^{\infty} \frac{\mu^{\circ(n)}}{c^{2 n}} \in \mathcal{P}_{+} .
$$

Additionally,

$$
\lambda=\mu^{2} \circ\left(\frac{\left(c^{2}-1\right)}{c^{2}} \delta_{0}+\frac{1}{c^{2}} \lambda\right)
$$

which yields

$$
\widehat{h}(\lambda)=\widehat{h^{2}}(\mu)\left(\frac{\left(c^{2}-1\right)}{c^{2}}+\frac{1}{c^{2}} \widehat{h}(\lambda)\right),
$$

$\mathbf{m}_{0}$-almost everywhere. The latter expression implies that $\mathbf{m}_{0}$-almost everywhere

$$
\widehat{h}(\lambda)\left(c^{2}-\widehat{h^{2}}(\mu)\right)=\left(c^{2}-1\right) \widehat{h^{2}}(\mu) .
$$

It follows from the above that, if $\left(c^{2}-\widehat{h^{2}}(\mu)(E)\right)=0$, then $\widehat{h^{2}}(\mu)(E)=0 \mathbf{m}_{0}$-almost everywhere and $\mathbf{m}_{0}(E)=0$. Thus, $\left(c^{2}-\widehat{h^{2}}(\mu)\right)>0 \mathbf{m}_{0}$-almost everywhere and

$$
\widehat{h}(\lambda)=\frac{\left(c^{2}-1\right) \widehat{h^{2}}(\mu)}{\left(c^{2}-\widehat{h^{2}}(\mu)\right)}
$$

$\mathbf{m}_{0}$-almost everywhere. The above expression contradicts equation 19 because $\widehat{h}(\lambda) \in \mathcal{F}_{+}$.

It would be natural to expect that the weak characteristic functions and the homomorphisms defined by the maximal ideals are strongly interrelated. For instance, it can be easily proved that the relationship

$$
h(\alpha)=\int \widehat{h}\left(\delta_{1}\right)(u) \alpha(d u), \alpha \in V_{0}
$$

produces a homomorphism of $\mathfrak{L}=\left(L_{1}\left(\mathbf{m}_{0}\right),+, \circ, \sim\right)$ corresponding to an ideal which belongs to $\mathfrak{M}_{+}^{s}$. We have already seen that this set is empty. Thus, the converse statement appears to be more interesting.

THEOREM 5.5. Each g.c. admits a weak characteristic function. A kernel $\mathfrak{M} \in \mathfrak{M}_{+}^{s}$ generates a weak characteristic function via the relationship (18).

As we have seen earlier, each weak characteristic function is uniquely defined via the kernel $\Phi_{\mathfrak{M}}\left(\delta_{1}, t\right)=\omega_{\mathfrak{M}}(t)$. It follows from theorem 5.3 that all weak characteristic functions are similar, that is

$$
\Phi_{\mathfrak{M}_{1}}(\mu, t)=\Phi_{\mathfrak{M}_{2}}(\mu, c t)
$$

for a constant $c>0$ and for all $\mu \in \mathcal{P}_{+}$. A similar conclusion can be drawn regarding the stable distributions. For this reason, in what follows, we will use the notation $\Phi(\mu, t)$ for a weak characteristic function, omitting the index $\mathfrak{M}$.

TheOrem 5.6. Suppose that $\varkappa(\circ)<\infty$. Then

1. $\lambda \in \boldsymbol{S}_{p}$ if and only if

$$
\Phi(\lambda, t)=\exp \left(-c t^{p}\right)
$$

holds $\mathbf{m}_{0}$-almost everywhere for some $c>0$ and $0<p \leq \varkappa(\circ)$; 
2. For any $0<p \leq \varkappa(\circ)$ all measures which belong to $\boldsymbol{S}_{p}$ are similar. This means that, if $\lambda_{1}, \lambda_{2} \in \boldsymbol{S}_{p}$, then $\lambda_{1}=T_{c} \lambda_{2}$ for some $c>0$.

As a direct consequence of the theorem, we obtain that all distributions which belong to $S \backslash S_{\varkappa}$ are equivalent to the Lebesgue measure. The set $S_{\varkappa}$ consists of the so-called characteristic measures arising as limit laws of axiom 5 for g.c. [51]. Each g.c. is completely defined by its characteristic exponent and characteristic measure, which can be formulated, more specifically, in the following form.

THEOREM 5.7. If $\circ$ and $\circ_{1}$ are general convolutions such that $\varkappa(\circ)=\varkappa\left(\circ_{1}\right)$ and the characteristic measures of $\circ$ and $\circ_{1}$ are similar, then the two general convolutions coincide.

The similarity of all weak characteristic functions allows to associate with every g.c. a subset $C(\circ) \subset \mathcal{F}_{+}$defined as: $f \in C(\circ)$ if and only if $f=\Phi(\mu, t) \mathbf{m}_{0}$-almost everywhere for some $\mu \in \mathcal{P}_{+}$. Evidently, this set does not depend upon the choice of a weak characteristic function.

Theorem 5.8. Let $f \in \mathcal{F}_{+}$, then $f \in C(\circ)$ if and only if the following two conditions hold:

1.

$$
\lim _{t \rightarrow 0} \frac{1}{t} \int_{0}^{t} f(u) d u=f(0)=1
$$

2. For any pair $\mu, \nu \in V_{0}$, the following inequality holds:

$$
\int f(u)(\mu \circ \mu)(d u) \int f(u)(\nu \circ \nu)(d u) \geq\left(\int f(u)(\mu \circ \nu)(d u)\right)^{2} .
$$

Moreover, if a o-quasi-invariant exists, then the g.c. is regular, which means that the kernel of the transform in 18 belongs to $\mathcal{C}_{+}$.

6. Realizations of regular g.c. It was mentioned in section 3.1 that the notion of g.c. was essentially based on the classical realizations presented in that section. However, finding new realizations is still a relevant problem. In this connection, it was suggested 22] to consider the so-called quasi-stable functions.

Definition 6.1. A function $f \in \mathcal{C}_{+}$is said to be quasi-stable if it has the Cauchy property. In other words, for every $a, b \in \mathbb{R}_{+}$, the function $f(a x) f(b x)$ belongs to the convex hull of the set $\left\{f(c x),: c \in \mathbb{R}_{+}\right\}$:

$$
f(a x) f(b x)=\int f(c x) \mu_{c(a, b)}(d c),
$$

where $\mu_{c(a, b)} \in \mathcal{P}_{+}$for every $a, b \in \mathbb{R}_{+}$.

THEOREM 6.2. A function $f \in \mathcal{C}_{+}$is a kernel of the characteristic function for a regular g.c. if and only if the following two conditions hold:

- $f$ is a quasi-stable function;

- $f(t)=1-t^{\kappa} L(t)$, where $\kappa>0$ and the function $L(t)$ is slowly varying at the origin; 
- there exists a measure $\sigma \in \mathcal{B}$ such that

$$
\varlimsup_{t \rightarrow \infty} \int f(x t) \sigma(d x)<1 .
$$

It should be noted that the second condition of the theorem was introduced by Bingham [4] with the aim of describing the domains of attraction of o-stable distributions. A slightly different standpoint, based on the notion of $\mathcal{B}$-stable probability distributions, was discussed by Urbanik [47.

Specifically, let $(\Omega, \mathcal{A}, \boldsymbol{P})$ be a probability space such that, for every sequence $\mu_{i} \in$ $\mathcal{P}(\mathbb{R}), i=1,2, \ldots$, there exists a sequence $\left\{X_{i}\right\}, i=1,2, \ldots$, of independent random variables on $(\Omega, \mathcal{A}, \boldsymbol{P})$ with probability distributions $\left\{\mu_{i}\right\}$. In fact, the variables are assumed to be symmetric. For a given sub- $\sigma$-algebra $\mathcal{B} \subset \mathcal{A}$, a random variable $X$ has a $\mathcal{B}$-stable distribution $\mu$ if, for each set of positive numbers $\left\{c_{i}\right\}, i=1, \ldots, n$, and each set of independent random variables $\left\{X_{i}\right\}, i=1, \ldots, n$, distributed according to $\mu$, there exist an independent non-negative $\mathcal{B}$-measurable random variable $C$ and a random variable $X_{0}$, distributed according to $\mu$, such that the variables $\sum_{i=1}^{n} c_{i} X_{i}$ and $C X_{0}$ are identically distributed:

$$
\sum_{i=1}^{n} c_{i} X_{i} \sim C X_{0} .
$$

Obviously, it is sufficient to establish this relationship only for $n=2$. For the trivial field $\mathcal{B}=\{\emptyset, \Omega\}$, a $\mathcal{B}$-stable distribution is a stable one in the classical meaning. Moreover, it is true for every finite field $\mathcal{B}$, as demonstrated in [47].

A multivariate generalization of the $\mathcal{B}$-stable distributions notion was suggested by Volkovich in 64, where random variables were considered as taking values in a Euclidean space. This construction can be reduced to the one-dimensional one by means of the well-known Cramer-Wold principle. It should be noted that this "multidimensional" point of view often provides visual geometric interpretation of the involved distributions. The characteristic function of a $\mathcal{B}$-stable distribution is, obviously, a quasi-stable function, where $\mu_{c(a, b)}$ from equation $(20)$, for $a, b>0$, is the distribution of the variable $C$ from equation (21) in the case of $n=2, c_{1}=a$, and $c_{2}=b$. The additional conditions of theorem 6.2 should be checked; yet, in contrast to other cases, this does not present a problem. In [34] the $\mathcal{B}$-stable distributions on a separable Banach space have been introduced. Here, they were named weak-stable distributions. Moreover, Misiewicz [33] defined infinitely divisible measures with respect to the generalized weak convolution. Jasiulis and Misiewicz [14] gave the Lévy-Khintchine representation for an infinitely divisible measure and defined $\mu$-stable measures in the sense of weak generalized convolution.

A regular g.c. is defined in the framework of the presented model by the operator

$$
Z^{a} f(b)=\int f(c) \mu_{c(a, b)}(d c), x, y \in \mathbb{R}_{+}, \quad f \in \mathcal{C}_{+} .
$$

All the classical realizations of regular generalized convolutions listed in section 3.1 can be obtained in such a way. Several new realizations discovered later have the following characteristic function kernels: 
- the Kucharczak and Urbanik convolutions

1.

$$
f_{n}(t)=\left\{\begin{array}{l}
(1-t)^{n} \text { for } 0 \leq t \leq 1 \\
0 \text { otherwise }
\end{array}\right.
$$

where $n \in \mathbb{N}$. Note that, for $n=1$, this convolution coincides with the Kendall convolution induced by the Kendall operation on random sets [19].

2 .

$$
f_{\alpha}(t)=\left\{\begin{array}{l}
\left(1-2^{(1+\alpha)\left[\log _{2} t\right]}-\right. \\
\left.-\left(2-2^{-\alpha}\right)\left(1-2^{\left[\log _{2} t\right]}\right) t^{\alpha}\right) \text { for } 0 \leq t \leq 1 \\
0 \text { otherwise }
\end{array}\right.
$$

where $0<\alpha<1$;

- the Volkovich convolutions defined for the parameter $0<\nu<\frac{1}{2}$ :

1.

$$
f_{\nu}(t)=\frac{t^{\nu} K_{\nu}(t)}{\Gamma(\nu) 2^{\nu-1}}
$$

where $K_{\nu}(t)$ is the Macdonald function of index $\nu$ [64];

2 .

$$
\omega_{\nu}(t)=\frac{t^{\nu}}{\Gamma(\nu) 2^{\nu}}\left(\cos (\nu \pi) K_{\nu}(t)-Y_{\nu}(t)\right),
$$

where $Y_{\nu}(t)$ is the Neyman function of index $\nu$ [73].

Another example of a non-regular convolution different from the $\infty$-convolution was constructed by Urbanik [53. Let $0<p<1$ be an arbitrary number and, for $\lambda \in \mathcal{P}_{+}$,

$$
\lambda(A)=(1-p) \delta_{1}+p \int_{A \cap[1, \infty]} u^{-2} d u .
$$

It is easy to show that

$$
\left(\lambda \otimes \delta_{0}\right) \circ\left(\lambda \otimes \delta_{0}\right)=\left(\lambda \otimes \delta_{0}\right)
$$

and, for $a \leq b, 0<b$,

$$
\left(\lambda \otimes \delta_{a}\right) \circ\left(\lambda \otimes \delta_{b}\right)=\lambda \otimes\left[\left(1-p \frac{a}{b}\right) \delta_{b}+p \frac{a}{b} T_{b} \sigma\right],
$$

where $\otimes$ is the "multiplicative convolution", $\circ$ is the $\infty$-convolution, and $\sigma$ has the density

$$
g(u)= \begin{cases}\frac{2 p}{(2 p-1) u^{3}}-\frac{1}{(2 p-1) u^{\frac{2-p}{1-p}}}, & p \neq \frac{1}{2}, 1 \leq u, \\ \frac{1+2 \log u}{u^{3}}, & p=\frac{1}{2}, 1 \leq u, \\ 0 & \text { otherwise. }\end{cases}
$$

For every $\nu, \mu \in \mathcal{P}_{+}$, there exists a uniquely defined measure $\rho(\nu, \mu)$ such that

$$
(\lambda \otimes \nu) \circ(\lambda \otimes \mu)=\lambda \otimes \rho(\nu, \mu) .
$$

Thus, the operation $(\nu, \mu) \rightarrow \rho(\nu, \mu)$ results in a non-regular g.c.

The notion of weakly stable random vectors has been introduced as a direct natural generalization of the $\mathcal{B}$-stable distribution notion. The essential assumption is that the 
random variable $X$ in (21) takes values in a measurable space, which is often associated with a Banach space endowed with the appropriate $\sigma$-algebra. Misiewicz, Oleszkiewicz and Urbanik 34 provided a full characterization of weakly stable distributions with a non-trivial discrete part and also substantially contributed to characterizing weakly stable distributions in a general case. Convolutions produced in this way were studied by Misiewicz [33, while Jasiulis and Misiewicz [13] described the relation between weakly stable and pseudo-isotropic distributions.

\section{Related models}

7.1. Stochastic convolution. An attempt to construct a general convolution model was presented by Volkovich [65], [66], 67], and [70]. The operation is defined here on the space $\mathcal{P}(\boldsymbol{X})$ of all probability measures on a separable complete metric space $\boldsymbol{X}$ endowed with the weak topology by means of the integral transform

$$
\Phi(\mu, m)=\int \omega(x, m) \mu(d x),
$$

where $\mu \in \mathcal{P}(\boldsymbol{X})$ and $m$ is an element of a separable complete metric space $\boldsymbol{M}$ such that $\omega(x, m)$ is a real bounded continuous function on $\boldsymbol{X} \times \boldsymbol{M}$.

Stochastic convolution $\circ$ is a binary operation on $\mathcal{P}(\boldsymbol{X})$ satisfying the following conditions:

- Each distribution $\mu$ is uniquely defined by its transform values $\Phi(\mu, m)$ for all $m \in \boldsymbol{M}$ and there is no closed subset of $\mathcal{M}$ with such a property;

- There exists $x_{0} \in \boldsymbol{X}$ such that $\omega\left(x_{0}, m\right)=1$ for all $m \in \mathcal{M}$;

- $\mu_{n} \rightarrow \mu$ if and only if

$$
\Phi\left(\mu_{n}, m\right) \rightarrow \Phi(\mu, m)
$$

for all $m \in \mathcal{M}$;

- $\mu_{3}=\mu_{1} \circ \mu_{2}$ if and only if

$$
\Phi\left(\mu_{3}, m\right)=\Phi\left(\mu_{1}, m\right) \Phi\left(\mu_{2}, m\right)
$$

for all $m \in \mathcal{M}$;

- Denote by $F\left(B_{0}\right)$ the set of all o-divisors of $B_{0} \subset \mathcal{P}(\boldsymbol{X})$. The set $F\left(B_{0}\right)$ is relatively compact if and only if $B_{0}$ is relatively compact.

The last condition plays a crucial role in the design of the model. Indeed, this is an analogue of the known shift-compact property of divisors introduced in [39]. This property of Urbanik's regular convolutions was established earlier by Bingham [4].

The notion of stochastic convolutions arises from the study of various operations on probability measures. The regular generalized convolution algebras provide a key example which yields good results on the basis of a promising axiomatic system. Other examples are: the above-mentioned Levitan convolution, the polynomial hypergroup convolutions of Lasser [24], 25], 26], and the convolutions of Askey and Gasper [3]. According to Hazod's MathSciNet review of [66], 
- further examples can be found in the context of (certain) abelian hypergroups in [6] and the literature cited therein;

- additionally, semigroups with stochastic convolution are related to the Hungarian semigroups, investigated by Székely and Ruzsa [41.

The general stochastic convolution model provides a common approach to the LévyKhintchine canonical representation of infinitely divisible probability measures and related negative definite functions. It also allows to characterize Gaussian distributions in terms of quadratic forms.

7.2. $V$-infinitely divisible laws. Another model which was prompted by the generalized convolutions notion was that of the $V$-infinitely divisible distributions suggested in [68], 71] and [73. Such measures arise in the problems of stochastic centering of the sums of real independent random variables and are related to the super-stable distributions of Zolotarev. Characterizations of probability laws by means of stochastic properties of linear statistics can be considered as another source for achieving similar goals. The similarity of integral representations of the ordinary characteristic function and logarithms of the characteristic function of regular infinitely divisible distributions and of $V$-infinitely divisible distributions suggests that all the above representations can be interpreted and analyzed from a common general point of view.

This can be, actually, done by means of external points of a compact convex set using Cauchy's representation theorem. Johansen [15] used Cauchy's theorem to obtain the representation of logarithms of the characteristic function of regular infinitely divisible distributions. (This result is presented, for example, in the well-known book of Linnik and Ostrovski [30, part 11).

The technique under consideration can also be applied to proving the classical Bochner theorem on the integral representation of the continuous positive definite function. A crucial component of Johansen's approach is the concept of the negative definite functions having properties similar to the properties of the ordinary positive definite functions. It should be noted that the relation between the two notions mentioned above was discussed in [2]. The representation for infinitely divisible distributions on commutative local compact groups was obtained by means of "the shift-compact property of the divisors set" [39] mentioned in section 7.1 .

Still another methodology based on the consideration of suitable test functions algebras is discussed in [76. Here the key point is a link from $V$-infinitely divisible distributions to the conditionally positive definite functions, which were introduced by Micchelli 32 and widely utilized in the approximation theory. Integral representations of conditionally positive definite functions can be used for studying the LévyKhintchine formulas and vice versa. So, a general description in the framework of the model is provided by means of the integral representation of the positive functional produced on an appropriate semi-normed ring. The Bochner's theorem, the LévyKhintchine formula and the representation of characteristic functions of $V$-infinitely divisible distributions can be considered as particular cases of this general representation. 
Definition 7.1. A distribution $\mu \in \mathcal{P}(\mathbb{R})$ is called $V$-infinitely divisible ( $V$-i.d.) if, for each natural $n$, there exists a distribution $\mu_{n}$ and a non-negative number $a_{n}$ such that

$$
e^{-a_{n}|t|^{2}} \Phi(\mu, t)=\Phi^{n}\left(\mu_{n}, t\right)
$$

for every $t \in \mathbb{R}$.

Here, $\Phi(\mu, t)$ is the regular characteristic function of $\mu \in \mathcal{P}(\mathbb{R})$.

Let us define the subsets $V_{s}, s>0$ of $V$-i.d. distributions with respect to the asymptotic behavior of a sequence $a_{n}$ in 22 as

$$
a_{n}=O(1) * n^{\alpha}, \alpha=1-\frac{1}{s+1}, n \rightarrow \infty .
$$

It is evident that

- $V_{0}$ is the set of regular infinitely divisible distributions;

- $V_{s} \subset V_{s+1}$ for every $s$.

It was proved in 68 and 73 that

- $\Phi(\mu, t) \neq 0$ for all $t \in \mathbb{R}$ if $\mu \in V_{s}$.

Some examples of the laws in the case of $\mu \in V_{s}$ can be obtained from Linnik's distributions with the characteristic functions

$$
\Phi(\mu, t)=\exp \left(-A|t|^{2}-|t|^{\gamma}\right),
$$

where $\left[\frac{\gamma}{2}\right]$ is an even number such that $\gamma$ is not an even number and $A$ is a positive sufficiently large constant. It is easy to see that $\mu \in V_{s}$ for

$$
s>\frac{\gamma}{2}-1
$$

$V$-i.d. distributions appear in problems related to characterizations by means of the stochastic properties of linear statistics (see, for example, [18, parts 2 and 5).

Let $\left\{X_{j}\right\}, j=1, \ldots, m$, be a sequence of independent identically distributed random real values which have a distribution $\mu$ and let $\left\{b_{j}\right\}, j=1, \ldots, 2 m$, be real numbers. We construct the forms

$$
L_{1}=\sum_{j=1}^{m} b_{j} X_{j} ; \quad L_{2}=\sum_{j=1}^{m} b_{m+j} X_{j}
$$

and consider the problem of the characterization probability laws employing the properties of the identical distribution of $L_{1}$ and $L_{2}$. If

$$
\max _{1 \leq j \leq m}\left|b_{j}\right| \neq \max _{1 \leq j \leq m}\left|b_{j+m}\right|
$$

then

- $\Phi(\mu, t) \neq 0$ for all $t \in \mathbb{R}$ and the function

$$
\omega(t)=\log \Phi(\mu, t)
$$

can be defined for all $t \in \mathbb{R}$;

- $\omega(t)=O\left(t^{\beta}\right)$ for $t \rightarrow \infty$ and some $\beta>0$;

- $\mu \in V_{s}$ for some $s>0$. 
We introduce the set $D_{0}$ of all test functions (i.e., infinitely differentiable complex functions having compact support) on the real line $\mathbb{R} . D_{k}, k>1$, are subsets of $D_{0}$ which satisfy the condition

$$
\int x^{j} h(x) d x=0
$$

for all $0 \leq j \leq k-1$. Note that, in this section, whenever integration limits are not specified, it is implied that the lower limit is $-\infty$ and the upper limit is $+\infty$. Denote by $\partial$ the differentiation operation on $D_{0}$ and by

$$
I(h)(x)=\int_{-\infty}^{x} h(u) d u
$$

the integration operation on $D_{0}$. It can be easily verified that

$$
\partial\left(D_{k}\right)=D_{k+1}, \quad I\left(D_{k}\right)=D_{k-1}, k>0 .
$$

Definition 7.2. A continuous function $f$ on $\mathbb{R}$ is called $m$-positive definite if

$$
\iint f(x-y) h(x) \overline{h(y)} d x d y \geq 0
$$

for each $h \in D_{m}$, where $\overline{h(y)}$ is the conjugate of $h(y)$.

It is well known that a 0-positive definite function is a regular positive definite function. By the famous Bochner theorem, the set of such functions coincides with the appropriate normalized set of all characteristic functions. For a 1-positive definite $f$, the function $(-f)$ is a negative definite function. Such a function arises [31] as the logarithm of the characteristic function of an infinitely divisible distribution. As mentioned above, in approximation theory, $m$-positive definite functions are referred to as conditionally positive definite functions.

THEOREM 7.3. If $\mu \in V_{s}$, then the function $f(t)=\log (\Phi(\mu, t))$ is an m-positive definite function for $m>s+1$.

Furthermore, we endow the set $D_{n}$, for an even $n=2 m$, with a semi-normed ring structure using the integral transform with the kernel

$$
K_{n}(x, t)=\frac{\exp (i t x)}{(i x)^{n}},
$$

where $x \neq 0, n>0$. Note that the technique of constructing a convolution-like operation is similar to the approach to constructing the stochastic convolution in section 7.1 . The integral transform is defined as

$$
\Psi_{n}(h, x)=\int K_{n}(x, t) h(t) d t=\frac{1}{(i x)^{n}} \int \exp (i t x) h(t) d t, h \in D_{n} .
$$

Let us introduce a binary operation on $D_{n}$ :

$$
(h \circ g)(t)=I^{(n)}(h * g),
$$

where "*" denotes the regular convolution of the functions $h, g \in D_{n}$ and $I^{(n)}$ is the $n^{t h}$ iteration of the integral operator $I$ on $D_{n}$. 
THEOREM 7.4. If $f=(h \circ g)$, then

- $f \in D_{n}$;

- $\Psi_{n}(f, x)=\Psi_{n}(h, x) \Psi_{n}(g, x)$ for all $x \in \mathbb{R}$.

Thus the triplet $\left(D_{n},+, \circ\right)$ yields a commutative ring. We can also define an involution operation $h^{\sim}(t)=\overline{h(-t)}$ and a family of semi-norms on this ring:

$$
\|h\|_{j}=\max _{|x| \leq j}\left|\Psi_{n}(h, x)\right|, \quad j=1,2,3, \ldots
$$

It is easy to see that:

- $\Psi_{n}\left(h^{\sim}, x\right)=\overline{\Psi_{n}(h, x)}$ for all $x \in \mathbb{R}$;

- $\left\|h^{\sim}\right\|_{j}=\|h\|_{j}$ for all $j=1,2,3, \ldots$;

- $\left\|\Psi_{n}\left(h^{\sim} \circ h, x\right)\right\|_{j}=\left\|\Psi_{n}(h, x)\right\|_{j}^{2}$ for all $j=1,2,3, \ldots$;

- The topology produced by the system of semi-norms $\|\cdot\|_{j}$ is not weaker than the weak-star topology induced by the set of all continuous functions on $\mathbb{R}$.

Denote by $R_{n}$ the closure of the symmetric algebra with the topology described above and build up a functional on $R_{n}$ defined by a continuous function $f$ :

$$
F_{2 m}(f)(h)=\int(-1)^{m} f(t) h(t) d t .
$$

THEOREM 7.5. If $f$ is an m-positive definite function, then the functional $F_{2 m}(f)(h)$ is a positive continuous functional on the algebra $\left(D_{2 m},+, \circ\right)$.

By this theorem, representations of positive functionals on semi-normed rings given in 7] can be employed. Thus

$$
F_{2 m}(f)(h)=\iint \frac{\exp (i t x)-\sum_{j=0}^{2 m-1}(i t x)^{j} / j !}{(i x)^{2 m}} \pi(d x) h(t) d t
$$

for a finite measure $\pi$ on $\mathbb{R}$, which results in

$$
f(t)=P_{2 m-1}(t)+\int \frac{\exp (i t x)-\sum_{j=0}^{2 m-1}(i t x)^{j} / j !}{x^{2 m}} \pi(d x),
$$

where $P_{2 m-1}(t)$ is a polynomial of degree $(2 m-1)$. The result 25 can be summarized in the following form:

THEOREM 7.6. Let $f$ be an m-positive definite function, then there exists a polynomial $P_{2 m-1}(t)$ of degree $(2 m-1)$ and a finite measure $\pi$ on $\mathbb{R}$ such that the representation 25) holds. The polynomial $P_{2 m-1}(t)$ and the measure $\pi$ are uniquely defined by $f$.

COROLlary 7.7. A continuous function $f$ on $\mathbb{R}$ satisfying the condition $f(0)=1$ is positive definite if and only if this function is the characteristic function of some distribution on $\mathbb{R}$.

In this case, $f$ is a 0 -positive definite function and, in view of $(25)$, we obtain $f(0)=1$ and

$$
f(t)=\int \exp (i t x) \pi(d x)
$$


where $\pi$ is a probability measure on $\mathbb{R}$. This is the Bochner representation of the positive definite functions.

Corollary 7.8. A probability measure $\mu$ is infinitely divisible if and only if

$$
\log \Phi(\mu, t)=i c t+\int \frac{(\exp (i t x)-1-i t x)}{x^{2}} \pi(d x),
$$

where $c$ is a real constant and $\pi$ is a finite measure on $\mathbb{R}$.

This corollary is, actually, a version of the Lévy-Khintchine formula for infinitely divisible distributions. As mentioned earlier, if a distribution $\mu$ is infinitely divisible, then $f(t)=\log (\Phi(\mu, t))$ is a 1-positive definite function. Taking into account that $f(0)=0$ and $f(-t)=\overline{f(t)}$, we obtain 26 from 25 .

An alternative approach, based on the relationship between $V$-infinitely divisible distributions and conditionally positive definite functions, was offered by Volkovich [75]. In the cited work, the generalized function theory is applied. Namely, according to theorem 7.3. the function $f(t)=\log (\Phi(\mu, t)), \mu \in V_{s}$, is an $m$-positive definite function for $m>s+1$. Substituting in $24 h=\partial^{(m-1)}(g)$, where $g \in D_{0}$, we can see that the function

$$
k(t)=(-1)^{m-1} f^{(2 m-2)}(t)
$$

is a positive definite generalized function, $f^{(2 m-2)}(t)$ being the generalized derivative of the function $f$. An integral representation of $f$ can be obtained by means of accurate iterative integration of the Bochner-Schwartz representation of the appropriate generalized function (see, for example, [60]). Other representations of this kind are also known in the generalized functions theory and one of them can be employed in our situation. Specifically, representation 25 can be interpreted as a consequence of the representation of conditionally appropriate positive definite generalized functions presented in [9].

8. Open problems. In this section, we briefly describe two open problems connected to the generalized convolution field.

The first problem is related to the extension of the generalized convolution notion to the distributions on the whole real line. Examples of such an operation can be found in [72], while another approach is presented in [34. Potential properties of operations on the whole real line have been also discussed in 12 .

The second problem is connected to the representativity of generalized convolutions. This problem had been indirectly discussed for a long time before it was finally formulated by Urbanik [48].

Let us consider two generalized convolutions, $\circ$ and $\circ^{\prime}$. The convolution $\circ$ is said to be representable in $\circ^{\prime}\left(\circ \prec \circ^{\prime}\right)$ if there exists a continuous non-trivial linear map

$$
h: \mathcal{P}_{+} \rightarrow \mathcal{P}_{+}
$$

with the following properties:

- $h(\mu \circ \nu)=h(\mu) \circ^{\prime} h(\nu)$;

- $h\left(T_{a} \mu\right)=T_{a} h(\mu)$. 
For regular g.c., the relationship $\circ \prec o^{\prime}$ implies that each characteristic function of $\circ$ is also a characteristic function of $o^{\prime}$, that is,

$$
\omega(t)=\int \widetilde{\omega}(t u) \mu(d u), \mu \in \mathcal{P}_{+},
$$

where $\omega$ and $\widetilde{\omega}$ are kernels of the characteristic functions of $\circ$ and $\circ^{\prime}$, respectively.

It is easy to see that the $\alpha$-convolution, $0<\alpha \leq 2$, is representable in the $(1,1)$ convolution due to the relationship

$$
e^{-t^{\alpha}}=\int_{-\infty}^{+\infty} \cos (u t) \gamma_{\alpha}(d u),
$$

where $\gamma_{\alpha}$ is a symmetric stable law with the power $0<\alpha \leq 2$. The same statement can be easily verified for all generalized convolutions produced by $B$-stable distributions. All known realizations of generalized convolutions can be reduced, by means of a power variable exchange, to convolutions representable in the $(1,1)$-convolution.

The open problem which arises from the above is whether there exists a realization of regular generalized convolutions which does not satisfy the latter property. In other words, the question is whether there exists a regular convolution which is non-representable in the $(1,1)$-convolution.

\section{References}

[1] K. Akbarkhojaev and V. Volkovich, On one class of realizations of generalized convolutions, Annals of Applied Mathematics, Tashkent Polytechnic Institute, 1981, 18-22 (in Russian)

[2] N. Akhiezer, The Classical Moment Problem and Some Related Questions in Analysis, Hafner Publishing Co., New York, 1965.

[3] R. Askey and G. Gasper, Convolution structures for Laguerre polynomials, J. D'Analyse Math. 37 (1977), 48-68.

[4] N. H. Bingham, Factorization theory and domains of attraction for generalized convolution algebras, Proc. Lond. Math. Soc. 23 (1971), 16-30.

[5] —, On a theorem of Kłosowska about generalized convolutions, Colloq. Math. 48 (1984), $117-125$.

[6] W. R. Bloom and H. Heyer, Continuity of convolution semigroups on hypergroups, J. Theor. Prob. 1 (1988), 271-286.

[7] S. Do Shin, On semi-normed rings with involution, Izv. Akad. Nauk USSR. 23 (1959), no. 4, 509-528 (in Russian).

[8] S. A. Grishechin, Nonlinear normalization in Urbanik's convolutions, J. Soviet Math. 47 (1989), 2699-2702.

[9] C. Jaeyoung, C. Soon-Yeong and K. Dohan, Bochner Schwartz type theorem for conditionally positive definite Fourier hyperfunctions, Positivity 7 (2003), 323-334.

[10] R. Jajte, Quasi-stable measures in generalized convolution algebras, Bull. Acad. Pol. Sci., Ser. Sci. Math. 24 (1976), 505-511.

[11] -, Quasi-stable measures in generalized convolution algebras II, Bull. Acad. Pol. Sci., Ser. Sci. Math. 25 (1977), 67-72. 
[12] B. H. Jasiulis, Limit property for regular and weak generalized convolution, J. Theor. Probab.

[13] B. H. Jasiulis and J. K. Misiewicz, On the connections between weakly stable and pseudoisotropic distributions, Stat. Probab. Lett. 78 (2008), 2751-2755.

[14] -, -, Weak Lévy-Khintchine representation for weak infinite divisibility, 2009, preprint.

[15] S. Johansen, An application of extreme-point methods to the representation of infinitely divisible distributions, Z. Wahrscheinlichkeitstheorie Verw. Geb. 5 (1966), 304-316.

[16] Z. J. Jurek, Some characterizations of the class L in generalized convolution algebras, Bull. Acad. Pol. Sci., Ser. Sci. Math. 29 (1981), 409-415.

[17] - Limit distributions in generalized convolution algebras, Probab. Math. Stat. 5 (1985), 113-135.

[18] A. Kagan, J. Linnik and C. R. Rao, Characterization Problems in Mathematical Statistics, Wiley, 1973.

[19] D. G. Kendall, Foundations of a theory of random sets, in: Stochastic Geom., Tribute Memory Rollo Davidson, 1974, 322-376.

[20] J. F. Kingman, Random walks with spherical symmetry, in: Colloquium on Combinatorial Methods in Probability Theory, 1962, 40-46.

[21] M. Kłosowska, On the domain of attraction for generalized convolution algebras, Rev. Roum. Math. Pures Appl. 22 (1977), 669-677.

[22] K. Kucharczak and K. Urbanik, Quasi-stable functions, Bull. Acad. Pol. Sci., Ser. Sci. Math. 22 (1974), 263-268.

[23] -, -, Transformations preserving weak stability, Bull. Acad. Pol. Sci., Math. 34 (1986), $475-486$.

[24] R. Lasser, Bochner theorems for hypergroups and their applications to orthogonal polynomial expansions, Appl. Theor. Prob. 37 (1983), 311-325.

[25] — Orthogonal polynomials and hypergroups, Rend. Mat. 3 (1983), 185-209.

[26] - On the Lévy-Hincin formula for commutative hypergroups, in: Probability Measures on Groupes VII, Lecture Notes in Mathematics 1064 (1984), 298-308.

[27] B. M. Levitan, Expansion in Fourier series and integrals with Bessel functions, Uspekhi Mat. Nauk 6 (1951), no. 2(42), 102-143 (in Russian).

[28] -, On a class of solutions of the Kolmogorov-Smoluchowski equation, Vestnik Leningrad. Univ. 15 (1960), no. 7, 81-115 (in Russian).

[29] Y. Linnik, Linear forms and statistical criteria, Ukr. Mat. Zh. 5 (1953), no. 2-3, 207-243, 247-290 (in Russian).

[30] Y. Linnik and J. Ostrovsky, Decomposition of Random Variables and Vectors, American Mathematical Society, 1977.

[31] E. Lukas, Characteristic Functions, Griffin, London, 1970.

[32] C. A. Micchelli, Interpolation of scattered data: distance matrices and conditionally positive definite functions, Constr. Approx. 2 (1986), 11-22.

[33] J. K. Misiewicz, Weak stability and generalized weak convolution for random vectors and stochastic processes, in: Institute of Mathematical Statistics Lecture Notes - Monograph Series 48 (2006), 109-118.

[34] J. K. Misiewicz, K. Oleszkiewicz and K. Urbanik, Classes of measures closed under mixing and convolution. Weak stability, Studia Math. 167 (2005), 195-213.

[35] M. A. Naimark, Normed Rings, Nauka, Moscow, 1968 (in Russian).

[36] Nguyen Van Thu, Semigroups in Urbanik convolution algebras, Acta Math. Vietnam. 14 (1989), 93-99. 
[37] I. D. Olshanetskii, Construction of a hypercomplex system with respect to the Urbanik algebra, Ukrain. Mat. Zh. 38 (1986), 801-804 (in Russian).

[38] I. V. Ostrovskii, Description of the class $I_{0}$ in a certain special semigroup of probability measures, Fiz.-Tehn. Inst. Nizkih Temperatur, Akad. Nauk Ukrain. SSR, Kharkov, 3-12 (in Russian).

[39] K. R. Parthasarathy, R. R. Ranga and S. R. S. Varadhan, On the category of indecomposable distributions on topological groups, Trans. Am. Math. Soc. 102 (1962), 200-217.

[40] - , - - , Probability distributions on locally compact abelian groups, Illinois J. Math. 7 (1963), 337-369.

[41] I. Z. Ruzsa, Infinite divisibility, Adv. Math., 69 (1988), 115-132.

[42] A. K. Smirnov, Some properties of distributions in K. Urbanik's algebras, in: Stability problems of stochastic models, J. Sov. Math. 40 (1988), 552-561.

[43] K. Urbanik, Generalized convolutions, Studia Math. 23 (1964), 21-245.

[44] -, A characterization of a class of convolutions, Colloq. Math. 1967, 239-249.

[45] -, Generalized convolutions II, Studia Math. 45, 1973, 57-70.

[46] - Extreme-point methods in probability theory, in: Lecture Notes in Math. 472, Springer, 1975, 169-194.

[47] -, Remarks on $\mathcal{B}$-stable probability distributions, Bull. Acad. Pol. Sci., Ser. Sci. Math. Astron. Phys. 22 (1976), 763-787.

[48] - Generalized convolutions III, Studia Math. 80 (1984), 167-189.

[49] - Joint probability distributions and commutability of observables, Demonstr. Math. 18 (1985), 31-41.

[50] —, Generalized convolutions, Uspekhi Mat. Nauk 40 (1985), no. 4 (244), 205-206.

[51] —, Generalized convolutions IV, Studia Math. 83 (1986), 57-95.

[52] - , A numerical constant associated with generalized convolutions, Colloq. Math. 51 (1987), 379-388.

[53] -, A counterexample on generalized convolutions, Colloq. Math. 54 (1987), 153-158.

[54] —, Generalized convolutions V, Studia Math. 91 (1988), 153-178.

[55] - Analytical methods in probability theory, in: Information Theory, Statistical Decision Functions, Random Processes, Trans. 10th Prague Conf., Vol. A, 1988, 151-163.

[56] —, Quasi-regular generalized convolutions, Colloq. Math. 55 (1988), 147-162.

[57] - Atoms of characteristic measures, Colloq. Math. 58 (1989), 125-129.

[58] -, Moments and generalized convolutions II, Probab. Math. Statist. 14 (1993), 1-9.

[59] -, Moments and generalized convolutions III, Probab. Math. Statist. 19 (1999), 153-169.

[60] V. S. Vladimirov, Generalized Functions in Mathematical Physics, Part 2, Nauka, Moscow, 1976 (in Russian).

[61] V. Volkovich, On an analytical description of Urbanik algebras, Izd. Akad. Nauk USSR, Ser. Fiz.-Mat. Nauk 24 (1979), no. 5, 12-17 (in Russian).

[62] -, On normalized rings generated by K. Urbanik's algebras, Theory of Probability and Its Applications 25 (1980), no. 2, 432-433 (in Russian)

[63] -, Normalized rings generated by generalized convolutions, in: Proc. Seminar in Problems of the Stability of Stochastic Models, 1980, 12-16 (in Russian).

[64] - Multidimensional $\mathcal{B}$-stable distributions and realizations of generalized convolutions, in: Stability Problems for Stochastic Models, 1984, 40-54 (in Russian).

[65] -, On algebras with stochastic convolution, in: 4th International Conference on Theory of Probability and Mathematical Statistics, Vilnius, Lithuania, 1985 (in Russian). 
[66] V. Volkovich, Infinitely divisible distributions in algebras with stochastic convolution, in: Stability Problems for Stochastic Models, Journal of Mathematical Sciences 40 (1988), 459-467.

[67] -, Quasi-regular stochastic convolutions, in: Stability Problems for Stochastic Models, Journal of Mathematical Sciences 47 (1989), 2685-2699.

[68] - On centering of probability distributions, Izvestya of the Academy of Sciences of the Uzbek SSR, Physical-Mathematical Series, 1990, no. 2, 3-9 (in Russian).

[69] - On stochastic convolution models, Probability Theory and Mathematical Statistics 2 (1990), 563-568 (in Russian).

[70] - On symmetric stochastic convolutions, Journal of Theoretical Probability 5 (1992), 417-430.

[71] - On integral representations of V-infinitely divisible distributions, in: 17th Seminar on Stability Problems for Stochastic Models, 1993.

[72] - Homogeneous models of convolution on the line, in: Stability Problems for Stochastic Models, Journal of Mathematical Sciences 69 (1994), 1134-1139.

[73] - On V-infinitely divisible distributions, Theory of Probability and Its Applications 3 (1995), 98-111.

[74] - Generalized Stochastic Convolution, Probability and Mathematical Statistics Encyclopedia, Publ. House of the Great Russian Encyclopedia, 1999.

[75] -, Remarks on proofs of the Lévy-Khinchine formulas from the point of view of the generalized functions, Journal of Mathematical Sciences 146 (2007), 6054-6058.

[76] Z. Volkovich, Z. Barzily and P. Sureanu, The Lévy-Khinchine representations and functional algebras of test functions, Journal of Pure and Applied Mathematics 25 (2005), 103-121.

[77] V. M. Zolotarev, One-Dimensional Stable Distributions, AMS Bookstore, 1986. 